\title{
Transfer after Dual $n$-Back Training Depends on Striatal Activation Change
}

\author{
ㄷTiina Salminen, ${ }^{1}$ Simone Kühn, ${ }^{2,3}$ Peter A. Frensch, ${ }^{1}$ and Torsten Schubert ${ }^{1,4}$ \\ ${ }^{1}$ Humboldt-Universität zu Berlin, Faculty of Life Sciences, Department of Psychology, 12489 Berlin, Germany, ${ }^{2}$ Max Planck Institute for Human \\ Development, Center for Lifespan Psychology, 14195 Berlin, Germany, ${ }^{3}$ University Clinic Hamburg-Eppendorf, Clinic and Policlinic for Psychiatry and \\ Psychotherapy, 20246 Hamburg, Germany, and ${ }^{4}$ Martin-Luther-Universität Halle-Wittenberg, Department of Psychology, 06108 Halle, Germany
}

The dual $n$-back working memory (WM) training paradigm (comprising auditory and visual stimuli) has gained much attention since studies have shown widespread transfer effects. By including a multimodal dual-task component, the task is demanding to the human cognitive system. We investigated whether dual $n$-back training improves general cognitive resources or a task-specific WM updating process in participants. We expected: (1) widespread transfer effects and the recruitment of a common neuronal network by the training and the transfer tasks and (2) narrower transfer results and that a common activation network alone would not produce transfer, but instead an activation focus on the striatum, which is associated with WM updating processes. The training group showed transfer to an untrained dual-modality WM updating task, but not to single-task versions of the training or the transfer task. They also showed diminished neuronal overlap between the training and the transfer task from pretest to posttest and an increase in striatal activation in both tasks. Furthermore, we found an association between the striatal activation increase and behavioral improvement. The control groups showed no transfer and no change in the amount of activation overlap or in striatal activation from pretest to posttest. We conclude that, instead of improving general cognitive resources (which would have required a transfer effect to all transfer tasks and that a frontal activation overlap between the tasks produced transfer), dual $n$-back training improved a task-specific process: WM updating of stimuli from two modalities.

Key words: dual $n$-back; fMRI; training; transfer; working memory

\section{Significance Statement}

The current study allows for a better understanding of the cognitive and neural effects of working memory (WM) training and transfer. It shows that dual $n$-back training mainly improves specific processes of WM updating, and this improvement leads to narrow transfer effects to tasks involving the same processes. On a neuronal level this is accompanied by increased neural activation in the striatum that is related to WM updating. The current findings challenge the view that dual $n$-back training provokes a general boosting of the WM system and of its neural underpinnings located in frontoparietal brain regions. Instead, the findings imply the relevance of task-specific brain regions which are involved in important cognitive processes during training and transfer tasks.

\section{Introduction}

Several studies have shown that working memory (WM) can be trained (Lustig et al., 2009; von Bastian and Oberauer, 2014; Au et

Received June 16, 2015; revised July 22, 2016; accepted Aug. 2, 2016.

Author contributions: T. Salminen, S.K., P.A.F., and T. Schubert designed research; T. Salminen performed research; T. Salminen analyzed data; T. Salminen, S.K., and T. Schubert wrote the paper.

This work was supported by the Deutsche Forschungsgemeinschaft (Grant Schu 1397/5-2 to T. Schubert) and the Humboldt Initiative Structured PhD program "Self-Regulation Dynamics across Adulthood and Old Age: Potentials and Limits" (Grant 2013-HISP-006 to T. Salminen).

The authors declare no competing financial interests.

Correspondence should be addressed to Tiina Salminen, Humboldt-Universität zu Berlin, Faculty of Life Sciences, Department of Psychology, Rudower Chaussee 18, 12489 Berlin, Germany. E-mail: tiina.salminen@hu-berlin.de.

DOI:10.1523/JNEUROSCI.2305-15.2016

Copyright $\odot 2016$ the authors $\quad 0270-6474 / 16 / 3610198-16 \$ 15.00 / 0$ al., 2015). Dual $n$-back is a WM training paradigm that includes two simultaneous $n$-back streams, which, in the important bimodal dual $n$-back version, consist of simultaneous auditory and visual $n$-back tasks. Therefore, the task entails WM and dual-task components. Dual $n$-back training may improve fluid intelligence (Jaeggi et al., 2008; but see Redick et al., 2013) and some studies have shown transfer from dual $n$-back training to executive functions and attention (Salminen et al., 2012; Lilienthal et al., 2013). These widespread effects suggest that the task's complexity might be optimal in boosting cognitive resources in a general way instead of improving merely narrow, task-specific processes.

By applying functional magnetic resonance imaging (fMRI), we investigated whether dual $n$-back training improves cognitive 


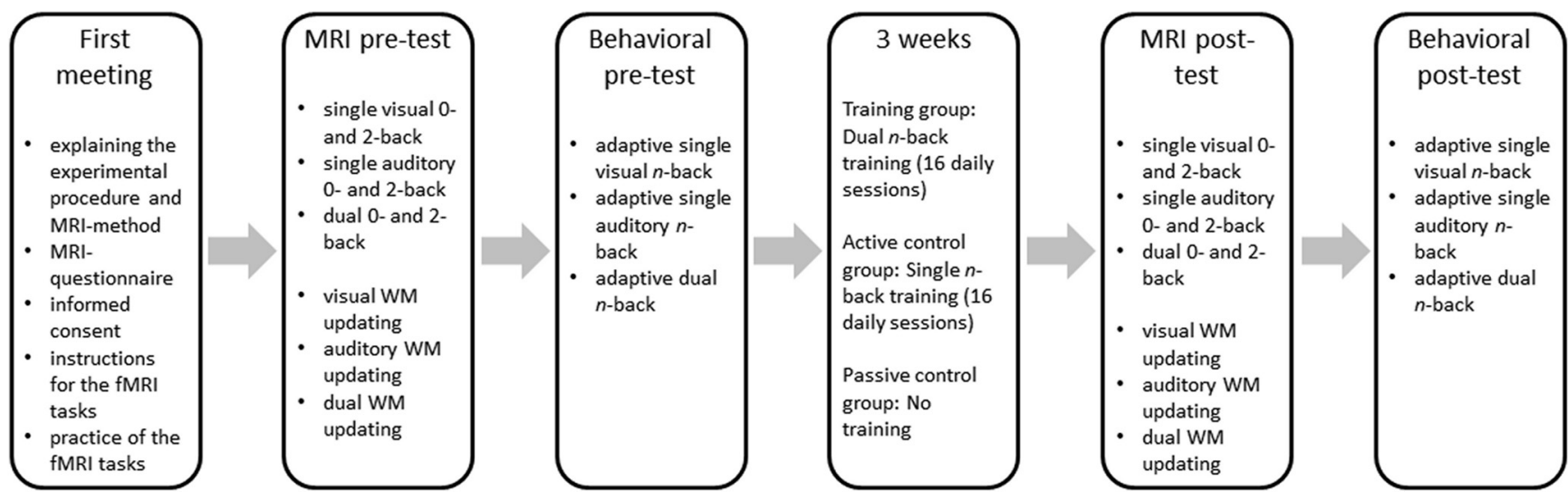

Figure 1. The study design.

resources in a general way or, alternatively, rather task-specific processes. The neural underpinnings of dual $n$-back training are still unsolved (but see Takeuchi et al., 2010). A bimodal $n$-back task presumably engages the prefrontal cortex (PFC) more strongly than a single-modality task because activations in the frontoparietal WM network correlate with both verbal and visuospatial WM (Klingberg, 2006; Takeuchi et al., 2011). Recent models place multimodal integration and dual-task coordination into the PFC, which is also responsible for other executive functions (Baddeley and Della Sala, 1996; Miller and Cohen, 2001).

Support for the hypothesis of a general improvement in cognitive resources comes from transfer effects after dual $n$-back training to tasks that conceptually only slightly overlap with the dual $n$-back (Jaeggi et al., 2008). Training of the general frontoparietal WM network should lead to improvements of the cognitive functions that use the same network (Klingberg, 2010). This general overlap hypothesis predicts that if training considerably engages the frontoparietal WM network and the transfer task generates a similar activation pattern, then (a) extensive training of this network produces a general boosting of cognitive resources, and (b) overlap of the neural activations promotes transfer (see also: Jonides, 2004).

An alternative hypothesis predicts that WM training effects transfer only if training improves specific cognitive processes required in both training and transfer tasks. Dahlin et al. (2008) found transfer after WM updating training to an $n$-back task that resembled the training task regarding updating processes, but not to a Stroop task that involved inhibition but not updating processes. By investigating neural activation patterns, the authors showed that an activation overlap by the three tasks in the frontoparietal WM network did not produce transfer; decisive was an overlapping activation by the training and the $n$-back task in the striatum, and a training-related increase in this overlap. Models ascribe WM updating processes to the striatum (O'Reilly and Frank, 2006; O'Reilly, 2006). Therefore, training had been process-specific (involving WM updating) and it did not lead to a general cognitive boosting (see also Kühn et al., 2013).

By testing near transfer effects (Karbach and Verhaeghen, 2014) to other WM tasks and the related neural changes, we aimed at elucidating the preconditions for transfer and the nature of training effects after dual $n$-back training. If dual $n$-back training boosts general cognitive resources, we expected (1) general improvements in several untrained single versions of the $n$-back (auditory-verbal and visuospatial), and transfer to untrained WM updating tasks (single auditory-verbal, single visuospatial, and dual-modality) after dual $n$-back training. Also, (2) neuronal overlap of the general frontoparietal network between the training and transfer task should then produce transfer.

Alternatively, if dual $n$-back training improves rather narrow task-specific processes, we expected transfer only to the dualmodality WM updating task, but not to single $n$-back and single WM updating tasks because the participants do not train to process these stimuli separately. Importantly, transfer should be associated with increased activation in an updating-process specific region, the striatum.

\section{Materials and Methods}

\section{Subjects}

Eighteen participants took part in the dual $n$-back training program (mean age 24.4 years, SD 4.0 years, range $20-32$ years, 6 male). Because we were specifically interested in the significance of the unique complexity of the task due to the dual-task component, an active control group was recruited to train on the single subtasks of the dual $n$-back-that is, the auditory-verbal (AV) and the visuospatial (VS) $n$-back tasks-separately. Accordingly, another 18 participants were assigned to the single $n$-back training program (mean age 24.1 years, SD 3.1 years, range 19-29 years, 4 male). As a third group, 18 participants (mean age 25.0 years, SD 4.0 years, range 19-33 years, 7 male) were assigned to a passive (nocontact) control group that did not undergo any training but attended only the pretest and posttest sessions. The groups did not differ significantly in age or sex distribution (both $p>0.54$ ). All participants were right-handed and reported normal or corrected-to-normal vision and normal hearing and received a compensation of $8 € / h$ for participation.

\section{Procedure}

Training took place on 16 consecutive days (excluding weekends); see Figure 1 for study design. The training group trained on the dual $n$-back task each day for $30 \mathrm{~min}$ and the active control group trained on each of their training task (the AV and the VS single $n$-back tasks) each day for 30 min Because the training time of the active control group was thus altogether $\sim 60$ min each day, the training group watched a $30 \mathrm{~min}$ documentary film at the end of each training session. This ensured that the participants of the training and the active control group spent the same amount of time in the laboratory environment. The participants were not informed about the purpose of the films.

Previous literature has shown significant training and transfer effects after 3 weeks or $8 \mathrm{~h}$ of training (Jaeggi et al., 2008; Klingberg, 2010). The training dose in the present study adds up to altogether $8 \mathrm{~h}$, and thus is in accordance with the recommendations of these studies. Before and after the training period, all participants attended an MRI-scanning session as well as behavioral pretests and posttests. The behavioral pretests and posttests were administered to compare behavioral changes between the groups, more specifically to assess the control groups' behavioral changes 
in the dual $n$-back task and the training group's behavioral changes in the single $n$-back tasks (in the scanner, the tasks included only the levels 0 and 2-back, which would have not provided a reliable assessment of improvements). The posttraining scanning session took place on the day directly after the final training day (equivalently for the passive control group who did not attend training). Before the first experimental session, each participant was informed about the experimental procedure. In addition, the participants filled out a questionnaire to ensure their suitability for MRI measurement, were informed about the MRI method, and signed an informed consent form. At the end of the first session, the participants were given the instructions for the fMRI tasks and, finally, they practiced each task for two runs. The participants in the training group and the active control group were at this point not yet informed about what their training task would be.

\section{Training tasks}

Dual n-back task. The dual $n$-back task consisted of simultaneously presented AV and VS $n$-back tasks (Buschkuehl et al., 2007). In the AV task, participants were presented with letters through headphones and, in the VS task, they saw blue squares in eight different locations on the computer screen. The letters and squares were presented simultaneously so that each stimulus appeared for $500 \mathrm{~ms}$, followed by a $2500 \mathrm{~ms}$ interstimulus interval. Each training session started on the $n$-back level 2 so that the participants were instructed to react whenever a currently presented item was the same as the item presented 2 steps back. However, in each training session, the task was adaptive so that, when the participant responded at least $90 \%$ correct in both tasks, he or she advanced to the next level (e.g., from 2-back to 3-back). If the participant responded 70\% or less correct during a run in either of the tasks, he or she fell to a lower level (e.g., from 3-back to 2-back), with the lowest possible level being 1 -back. In any other case, the $n$-back level remained constant. Participants received feedback on their performance after each block and were informed about the $n$-back level of the next run. Each training session comprised 20 runs and each run included $20+n$ trials (e.g., a 2-back run consisted of 22 trials). Participants were instructed to press the key "L" with the right index finger for the AV targets and the key " $A$ " with their left index finger for the VS targets. The task was self-paced and participants could start a new block by pressing the spacebar. In the behavioral pretest and posttest (outside the scanner), the task was the same as during training with 12 runs.

Single $\mathrm{n}$-back tasks. The single $n$-back tasks were the component (AV and VS) tasks of the dual $n$-back task. The stimuli, response keys and mappings, starting $n$-back level, feedback, and rules of adaptiveness were equal to the dual $n$-back task. However, the active control group never practiced the AV and VS task simultaneously, but rather, in each training session, the tasks were trained as single tasks with 20 runs of each task. The order of the AV and the VS task was counterbalanced so that every other training session started with one of the tasks and every other session with the other task. In the behavioral pretest and posttest (outside of the scanner), the task was the same as during training with six runs in the AV and the VS task each.

\section{fMRI tasks}

In the scanner as the participants lay on their backs, the two response devices (one for each hand) were placed on their legs. Both devices included four response buttons that were placed horizontally next to each other. In the $n$-back task, only the innermost buttons were needed; that is, the innermost button of the left response device for the left forefinger and the innermost button of the right response device for the right forefinger. In the WM updating transfer tasks, all buttons were required so that there was one button for each finger except for the thumbs.

$\mathrm{n}$-back tasks. The stimuli in the fMRI $n$-back tasks were the same as in the training tasks. However, the tasks were not adaptive and the $n$-back levels were 0 -back and 2-back in all versions of the $n$-back task: single AV, single VS, and the dual $n$-back. Note that the administration of nonadaptive versions in the fMRI sessions ensured that an equally effective memory load of 0 or 2 items could be associated with the resulting fMRI activity across subjects. In the 0-back task, the participants were instructed to respond to a certain letter (AV) and to a certain position of the blue square (VS). There were six blocks of $10 n$-back runs of each task (an $n$-back run refers to one condition in the task, e.g., VS 2-back) and each run included 10 trials. Therefore, there were altogether 18 task blocks. The blocks were presented in random order with a task instruction screen presented before each block. In addition, there were four baseline blocks during which the participants were presented with a fixation cross in the middle of the screen for $30 \mathrm{~s}$. Responses were given for targets only using the right index finger for the AV stimuli and the left index finger for the VS stimuli (in accordance with the training task).

WM updating transfer tasks. The WM updating transfer task was based on the letter memory task by Miyake et al. (2000). It consisted of three blocks: an AV, a VS, and a dual-modality block in which the AV and VS stimuli were presented simultaneously. The order of the AV and the VS blocks was counterbalanced, half of the participants started with the AV block and the other with the VS block; all participants performed the dual-modality block as last. For each participant, the order of the AV and the VS block was the same at pretest and at posttest. All blocks included nine sequences of items and the lengths of the sequences varied randomly so that a sequence could comprise $7,9,11,13$, or 15 items. The participants were unaware of the length of the ongoing sequence and they were asked to constantly update the contents of their WM. In the AV task, the participants were presented with the numbers 1,2,3, and 4 through headphones sequentially in a random order. After each sequence, the participant was asked to report the last four numbers in the correct order, starting from the fourth last and ending with the last presented item. The participants were asked to respond as correctly as possible, keeping in mind that they had $8 \mathrm{~s}$ after the question to report the last 4 items and then the task would continue automatically. The participants used their right hand to give responses: index finger for 1, middle finger for 2, ring finger for 3 , and little finger for 4 . After each response phase, a fixation cross was presented for $10 \mathrm{~s}$ and this time was used as a baseline period in the fMRI analyses. The procedure of the VS block was similar to the AV block, but the stimuli consisted of black bars presented one by one in four different locations from bottom to top on the screen. The participants used their left hand for the responses: the index finger for a bar presented in the lowermost part of the screen, the middle finger for a bar presented slightly below the midline of the screen, the ring finger for a bar presented slightly above the screen, and the little finger for a bar presented on the uppermost part of the screen.

The dual $n$-back training task and the WM updating transfer tasks were characterized by a number of similarities and differences. All tasks engaged WM updating processes, so this process was shared by the training and the transfer tasks. However, the task conditions differed with respect to stimuli (squares and letter in the $n$-back tasks vs bars and digits in the updating transfer tasks) and task rules (single-modality vs dualmodality; recognition of previously presented items in the $n$-back tasks vs recollection of items in the updating transfer tasks). With these characteristics, the tasks were near transfer tasks by definition of Karbach and Kray (2009; see also Karbach and Verhaeghen, 2014) and this allowed us to approach our research question by controlling the degree of similarity between the training and transfer tasks.

\section{fMRI data acquisition}

Images were acquired with a 3.0 T Siemens Magnetom Trio -scanner using a 12-channel radiofrequency head coil. First, high-resolution T1weighted 3D MPRAGE structural volumes were collected (repetition time $=2500 \mathrm{~ms}$, echo time $=4.77 \mathrm{~ms}$, acquisition matrix $=256 \times 256 \times$ 176 , flip angle $=7^{\circ}$, voxel size $=1 \times 1 \times 1 \mathrm{~mm}^{3}$ ). Subsequently, for BOLD signal acquisition during the fMRI tasks, whole-brain functional images were collected using a $\mathrm{T} 2{ }^{\star}$-weighted echoplanar imaging sequence (repetition time $=2000 \mathrm{~ms}$, echo time $=30 \mathrm{~ms}$, image matrix $=$ $64 \times 64$, field of view $=216 \mathrm{~mm}$, flip angle $=80^{\circ}$, slice thickness $=3.0$ $\mathrm{mm}$, distance factor $=20 \%$, voxel size $=3 \times 3 \times 3 \mathrm{~mm}^{3}, 36$ axial slices, using GRAPPA). Images were aligned to the anterior-posterior commissure line.

All participants first completed in the scanner the $n$-back task divided into two blocks (both blocks including single and dual $n$-back runs of each level of $n$ in a random order), after which the WM updating tasks were completed. 
Table 1. Pretest and posttest performance and the effect sizes for pretest and posttest comparisons in the training group and the active and passive control groups in each task

\begin{tabular}{|c|c|c|c|c|c|c|c|c|c|}
\hline \multirow[b]{2}{*}{ Task } & \multicolumn{3}{|c|}{ Training group } & \multicolumn{3}{|c|}{ Active control group } & \multicolumn{3}{|c|}{ Passive control group } \\
\hline & Pretest & Posttest & $d$ & Pretest & Posttest & $d$ & Pretest & Posttest & $d$ \\
\hline Dual $n$-back in mean achieved $n$-back level ${ }^{a}$ & $2.68(0.53)$ & $4.74(1.05)$ & 2.48 & $2.45(0.56)$ & $3.67(0.83)$ & 1.72 & $2.59(0.73)$ & $2.98(0.87)$ & 0.49 \\
\hline Auditory & $3.32(0.48)$ & $4.18(0.47)$ & 1.81 & $3.06(0.61)$ & $4.27(0.36)$ & 2.42 & $3.32(0.56)$ & $3.53(0.50)$ & 0.40 \\
\hline Visual & $3.62(0.43)$ & $4.17(0.36)$ & 1.39 & $3.31(0.56)$ & $4.23(0.46)$ & 1.80 & $3.46(0.62)$ & $3.64(0.48)$ & 0.32 \\
\hline Dual WM updating ${ }^{b}$ performance in trials correct & $2.06(1.35)$ & $3.67(1.75)$ & 1.03 & $1.50(1.15)$ & $1.89(2.05)$ & 0.23 & $1.35(1.32)$ & $1.71(2.11)$ & 0.20 \\
\hline \multicolumn{10}{|l|}{ Single WM updating ${ }^{b}$ performance in trials correct } \\
\hline
\end{tabular}

Data are shown as means (SD).

${ }^{a}$ Training group includes 17 participants in the dual $n$-back task.

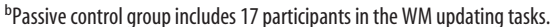

\section{fMRI data analyses}

Preprocessing. fMRI analyses were performed using SPM8 software (Wellcome Trust Centre for Neuroimaging, Institute of Neurology, University College London, UK; http://www.fil.ion.ucl.ac.uk/spm/). Each functional dataset was corrected for slice timing, realigned and warped into the standard MNI space (interpolated spatial resolution $3 \times 3 \times 3$ $\mathrm{mm}$ ), coregistered to the anatomical image, and finally smoothed using an $8 \mathrm{~mm}$ FWHM Gaussian filter kernel.

Statistical analyses. First, in the single-subject level analysis, each task ( $n$-back, WM updating) was modeled and convolved with the canonical hemodynamic response function. In the $n$-back task, we modeled each task block for pretest and posttest separately (AV 0-back, VS 0-back, dual 0-back, AV 2-back, VS 2-back, and dual 2-back), excluding the task instruction screen preceding each block. The duration of each task block was $15,000 \mathrm{~ms}$. In the WM updating task, we modeled only the updating phase of each sequence (and not the response phase; see also: Dahlin et al., 2008) separately for each task block (AV, VS, and dual) and for pretest and posttest. The durations of sequences with 7, 9, 11, 13, and 15 items were $11,000,14,000,17,000,20,000$, and $23,000 \mathrm{~ms}$, respectively. The design matrices of both tasks also included the six movement regressors. Subsequently, in a group-level analysis, contrasts in each task were computed separately for pretest and posttest. In the $n$-back task, we computed the contrast $[(2$-back $)-(0$-back $)]$ in each modality (AV, VS, and dual). In the WM updating task, we contrasted the updating phase against an implicit baseline.

For all analyses, a significant activation was reported at an overall significance level of $p<0.05$, corrected for multiple comparisons by a combination of a minimum cluster size of 22 and an intensity threshold of $p<0.001$. These parameters were derived using a Monte Carlo simulation with the AlphaSim tool of the AFNI software package (Ward, 2000).

To test the general overlap hypothesis of transfer, we aimed at identifying brain regions that are commonly activated by the training task and any task to which there was transfer. Because we found transfer in the training group only to the dual WM updating task (other groups showed no transfer; see Results), we analyzed overlap between the dual $n$-back task and the dual WM updating task. For that purpose, we conducted a conjunction analysis for Contrast 1: [(dual 2-back) - (dual 0-back)] and Contrast 2: (dual WM updating - baseline) (Nichols et al., 2005). A voxel was reported active only when it was significant for both contrasts, Contrast 1 and Contrast 2. In other words, we tested for a rejection of the conjunction null hypothesis: voxel (not activated in Contrast 1) or (not activated in Contrast 2). The conjunction analysis was performed for each group separately for pretest and posttest.

To foreshadow, the conjunction analysis revealed that all groups showed overlapping activations between the two tasks at pretest (see Results). Notably, whereas the active and passive control groups showed similar overlap at posttest as at pretest, the training group showed less overlap at posttest than at pretest. Because the training group was the only group that showed a transfer effect, the results of the conjunction analyses imply that an overlap in the general frontoparietal network alone does not produce transfer. Therefore, we tested our hypothesis about the role of training-related activation changes (i.e., increases or decreases) in one or more brain regions that are associated with specific cognitive processes. In particular, we looked at specific training-related changes separately for the dual $n$-back task and the dual WM updating task to investigate whether there are activation increases in the striatum after dual $n$-back training (see also Dahlin et al., 2008).

In the $n$-back task, this analysis included [(dual 2-back pretest - dual 0-back pretest) - (dual 2-back posttest - dual 0-back posttest)] for activation decreases after training, and [(dual 2-back posttest - dual 0-back posttest) - (dual 2-back pretest - dual 0-back pretest)] for activation increases after training. In the WM updating task, the analysis included [(dual updating posttest - baseline posttest) - (dual updating pretest - baseline pretest)] for activation increases after training and [(dual updating pretest - baseline pretest) - (dual updating posttest baseline posttest)] for activation decreases after training.

\section{Results}

\section{Behavioral results}

Due to a technical failure, the data of one participant from the training group were not recorded in the pretest session of the dual $n$-back task and the data of one participant from the passive control group were not recorded in the pretest session of the WM updating tasks. We excluded the data of these participants from the analyses of the corresponding tasks.

Means and SDs for each task and group at pretest and posttest, along with effect sizes (Cohen's $d$ ) for group-specific pretestposttest comparisons are presented in Table 1. First, a multivariate ANOVA (MANOVA, Pillai's trace) was conducted, with group (training vs active control vs passive control) as a betweensubjects factor and session (pretest vs posttest) as a withinsubjects factor on the data of all tasks as dependent variables (i.e., the mean level of $n$ in the behavioral $n$-back tasks and the number of correctly reported items in the WM updating tasks. This analysis revealed significant main effects of Session $\left(F_{(6,44)}=56.20\right.$, $\left.p<0.001, \eta_{p}^{2}=0.89\right)$ and group $\left(F_{(12,90)}=2.11, p<0.05, \eta_{p}^{2}=\right.$ $0.22)$. Importantly, the Group $\times$ Session interaction was significant $\left(F_{(12,90)}=6.45, p<0.001, \eta_{p}^{2}=0.46\right)$, which indicated that there were reliable group-specific performance changes from pretest to posttest.

Next, we report the follow-up analyses for each task. In the behavioral data of the $n$-back tasks, we analyzed the mean achieved $n$-back level of the adaptive version of the $n$-back task in the behavioral pretest and posttest. For the WM updating task, we analyzed the number of correctly reported four-item sequences in each task (AV, VS, and dual). Separate mixed-design ANOVAs for the $n$-back tasks and for the WM updating tasks were conducted with the factors group (training vs active control vs passive control) and session (pretest vs posttest). 

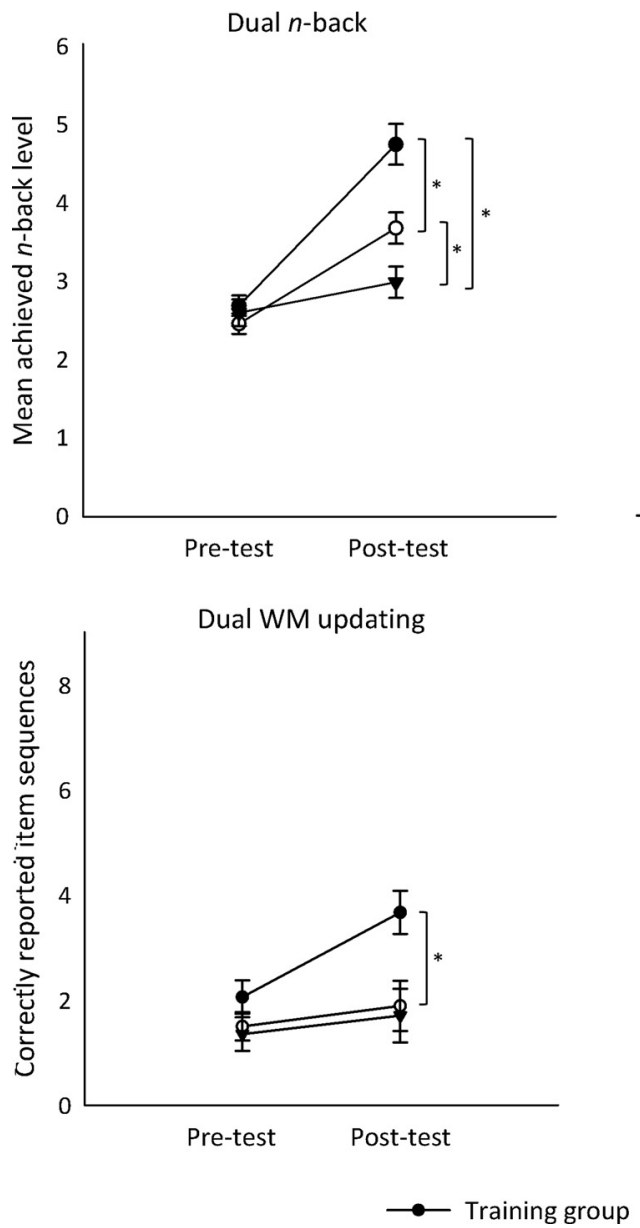

Auditory single $n$-back
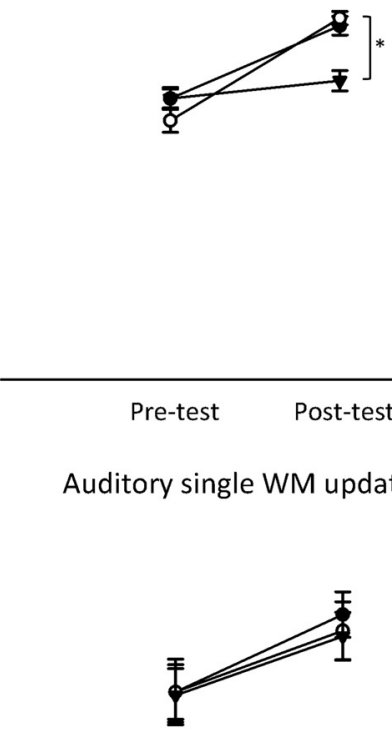

Visual single $n$-back

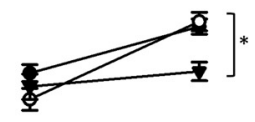

Pre-test

Auditory single WM updating

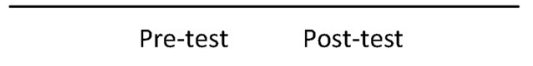

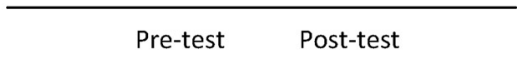

Visual single WM updating

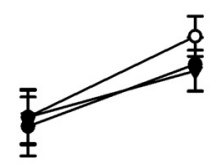

Figure 2. Top, Improvement in the performance of all groups from pretest and posttest in the dual $n$-back task (left), the auditory single $n$-back task (middle), and the visual single $n$-back task (right). For each session, the mean $n$-back level is presented. Error bars indicate SEM. An asterisk indicates a significant difference between the groups. Bottom, Improvement in the performance of all groups from pretest and posttest in the dual WM updating task (left), the auditory single WM updating task (middle), and the visual single WM updating task (right). For each session, the number of correctly reported item sequences is presented. Error bars indicate SEM. An asterisk indicates a significant difference between the groups.

Training tasks

Figure 2 (top) depicts the mean achieved $n$-back level at pretest and posttest for each group (training, active control, passive control) and task (dual $n$-back, AV single $n$-back, VS single $n$-back) separately.

Dual n-back

The main effects of group $\left(F_{(2,50)}=8.02, p<005, \eta_{p}^{2}=0.24\right)$ and session $\left(F_{(1,50)}=162.58, p<0.001, \eta_{p}^{2}=0.77\right)$ reached significance just as the important group $\times$ session interaction $\left(F_{(2,50)}=\right.$ 25.06, $\left.p<0.001, \eta_{p}^{2}=0.50\right)$. The latter indicates a differential change from pretest to posttest between the groups, with the training group showing the strongest improvement $(M=2.06)$, followed by the active control group $(M=1.21)$ and followed by the passive control group ( $M=0.39)$ (Fig. 2). Follow-up analyses revealed that, whereas the groups did not differ in their pretest performance $(p=0.85)$, all comparisons showed significant differences at posttest (training vs active control: $p<0.005$; training vs passive control: $p<0.001$; active control vs passive control: $p<0.05$ ). As expected, the training group showed most benefit from training by reaching the highest level of $n$-back $(M=4.75)$ at posttest. However, the active control group also benefited from the intervention because, at posttest, this group reached a significantly higher level of $n$ than the passive control group $(M=3.67$ and $M=2.59$, respectively).
Single n-back

In the analysis of the AV single $n$-back task, the main effect of session was significant $\left(F_{(1,51)}=104.48, p<0.001, \eta_{p}^{2}=0.67\right)$. In addition, the interaction between the factors group and session reached significance $\left(F_{(2,51)}=15.40, p<0.001, \eta_{p}^{2}=0.38\right)$. Therefore, in this task, there were also differences in the amount of improvement between the groups. However, here, the training and the active control group showed an equal improvement ( $M=0.85$ and $M=1.20$, respectively), whereas the passive control group showed less change from pretest to posttest $(M=$ 0.21 ). This observation was confirmed with follow-up analyses that showed that there was no difference in the posttest performance of the training and the active control group ( $p=0.53)$, but the passive control group differed significantly from the other two groups (both $p \leq .001$ ) (Fig. 2). There were no differences between the groups at pretest $(p=0.28)$.

The results for the VS single $n$-back task were similar. The main effect of session was significant $\left(F_{(1,51)}, p<0.001, \eta_{p}^{2}=\right.$ $0.49)$, as were the main effect of group $\left(F_{(2,51)}=3.45, p<0.05\right.$, $\left.\eta_{p}^{2}=0.12\right)$ and the group $\times$ session interaction $\left(F_{(2,51)}=7.50\right.$, $\left.p<0.005, \eta_{p}^{2}=0.23\right)$. Again, the training and the active control group improved equally from pretest to posttest ( $M=0.55$ and $M=0.93$, respectively), whereas the passive control group showed a smaller change $(M=0.18)$. Indeed, at 
posttest, there was no difference in the mean achieved $n$-back level between the training group and the active control group ( $p=0.66$ ) (Fig. 2). Conversely, the passive control group differed significantly from both of the other two groups (both $p \leq .005)$. There were no differences between the groups at pretest $(p=0.23)$.

\section{WM updating transfer tasks}

Figure 2 (bottom) depicts the mean number of correctly reported item sequences at pretest and posttest for each group (training, active control, passive control) and task (dual WM updating, AV single WM updating, VS single WM updating) separately.

\section{Dual WM updating}

All participants improved their performance from pretest to posttest, as reflected by the significant main effect of session $\left(F_{(1,50)}=12.88, p<0.01, \eta_{p}^{2}=0.21\right)$. The main effect of group was significant $\left(F_{(2,50)}=4.39, p<0.05, \eta_{p}^{2}=0.15\right)$, as well as the important group $\times$ session interaction, $F_{(2,50)}=3.61, p=0.05$, $\eta_{p}^{2}=0.13$. To gain more insight into group-specific performance changes, the pretest and posttest performances were compared within each group separately. The comparison revealed that, whereas the training group improved from pretest to posttest significantly $\left(t_{(17)}=-4.68, p<0.001\right.$, Cohen's $\left.d=1.03\right)$, there were no performance changes between the sessions in the active or the passive control groups (both $p>0.39$; Fig. 2). At pretest, there were no differences between the groups $(p=0.23)$. Altogether, these results point to robust transfer effects to the dual WM updating task after training on the dual $n$-back task.

\section{Single WM updating}

In the AV single WM updating task, the main effect of session reached significance $\left(F_{(1,50)}=26.01, p<0.001, \eta_{p}^{2}=0.28\right)$, reflecting the observation that all groups showed a slight improvement from pretest $(M=4.94)$ to posttest $(M=6.23)$ (Fig. 2). There were no other significant findings (all $p>0.88$ ).

The results in the VS single WM updating were similar. Across all groups, there was a significant improvement from pretest $(M=4.06)$ to posttest $(M=5.16)\left(F_{(1,50)}=10.77, p<0.01, \eta_{p}^{2}=\right.$ 0.18 ; Fig. 2). Other effects were not significant (all $p>0.71$ ).

\section{Imaging results}

Commonalities of activation between training and transfer tasks

To investigate commonalities (i.e., an overlap) between the activations in the dual $n$-back and the dual WM updating task (general overlap hypothesis), we conducted a conjunction analysis between the dual 2-back task [contrast of (dual 2-back - dual 0 -back)] and the dual WM updating task [contrast of (dual WM updating - baseline)]. The general overlap hypothesis of transfer predicts that we should see overlapping frontoparietal activations between the training and the transfer task in the training group and, by the time of posttest, this overlap should be the strongest for the training group that has shown most transfer compared with the other two groups. Therefore, we primarily focused the analyses on those tasks between which we found transfer, the dual $n$-back task and the dual WM updating task. We investigated the neural overlap between the two tasks at pretest and at posttest for each group separately. At pretest, we observed in all groups common frontoparietal activation along with joint activations in the striatum (Tables 2, 3, 4, Fig. 3). These activations are consistent with previous reports on WM-related neural networks, including regions in the frontal (BAs 8, 9, 10,44, and 46) and in the parietal cortices. The subcortical activations reflect the involvement of these regions in WM updating processes as postulated by previous studies and models (O'Reilly and Frank, 2006; O'Reilly, 2006; Dahlin et al., 2008). Most importantly, at posttest, the training group showed clearly fewer overlapping regions between the two tasks, especially in the frontal cortex, where some overlapping regions remained only mainly in BAs 6 and 44 (Fig. 3, Table 2). In particular, such reduced overlap of activation may be related to more sparse and sharper neural representation of the relevant task processes in the dual $n$-back task leading to a decrease of the activation overlap with the dual-updating task after training (van Turennout et al., 2000). There was still also an overlap in the left striatum. With a more lenient threshold of $p<0.05$ (uncorrected; keeping the cluster size of $>22$ voxels), we additionally observed overlap in the right striatum.

In contrast, for the active and passive control groups, the analysis revealed that the number of overlapping neural regions either stayed constant or even increased from pretest to posttest (Fig. 3, Tables 3, 4). That is, both control groups still showed activation overlap in similar regions of the PFC at posttest as at pretest, as well as in the parietal cortex and in the bilateral striatum.

In sum, all groups showed neuronal overlap between the training and the transfer task in the frontoparietal network at pretest. At posttest, whereas both control groups still showed overlap between the two tasks in this network, in the training group, the overlapping regions had diminished. As can be seen in Figure 3, this diminishment was especially expressed in regions of the frontal cortex, that is, anteriorly to the central sulcus. To justify statistically the visual impression of different patterns of overlapping frontal activations between the three groups as resulting from training, we conducted the following analysis. First, we extracted the number of overlapping voxels in the frontal cortex during performance of the two task types in the pretest and posttest data for each participant. A one-way ANCOVA between the three groups on the number of overlapping voxels at posttest, controlling for the amount of overlapping voxels at pretest, yielded a significant result $\left(F_{(2,50)}=\right.$ 3.76, $p<0.05, \eta_{p}^{2}=0.13$ ). Importantly, because the training group was the only group that showed transfer between the dual $n$-back and the dual WM updating task, these results imply that the common activation of a frontoparietal network is not a decisive precondition for transfer between the two tasks.

This claim is also supported when analyzing the pattern of activation overlap for tasks other than the dual $n$-back and dual WM updating tasks, namely the single versions of the $n$-back and the WM updating tasks, for which we did not find transfer effects. With this subordinate analysis, we found a similar pattern of activation overlap in the frontoparietal network for all three groups at pretest (Fig. 4), which is similar to the situation with the dual-task versions; moreover, the overlap pattern changed in a manner similar to the dual-task versions as a result of training. In particular, we found that the amount of overlap decreased from pretest to posttest significantly more for the subjects of the active control group who trained the single $n$-back tasks compared with the other two groups. This is supported by the results of corresponding ANCOVAs between the three groups on the number of overlapping voxels at posttest with the number of overlapping voxels at pretest as covariate $\left(\mathrm{AV}: F_{(2,50)}=4.58, p<0.05, \eta_{p}^{2}=\right.$ 0.16 ; VS: $\left.F_{(2,50)}=11.20, p<0.001, \eta_{p}^{2}=0.31\right)$.

Notably, the participants of the active control group did not show transfer effects from the $n$-back task situations to any of the 
Table 2. Regions in which overlapping activations were observed between the training task [(dual 2-back) - (dual 0-back)] and the transfer task (dual WM updating baseline) in the training group at pretest and at posttest

\begin{tabular}{|c|c|c|c|c|c|c|c|c|c|c|c|c|c|c|c|}
\hline \multicolumn{8}{|l|}{ Pretest } & \multicolumn{8}{|l|}{ Posttest } \\
\hline \multirow[b]{2}{*}{ Area } & \multirow[b]{2}{*}{ Location } & \multirow[b]{2}{*}{ BA } & \multicolumn{3}{|c|}{ Peak Talairach coordinates } & \multirow[b]{2}{*}{$t$} & \multirow[b]{2}{*}{ CS } & \multirow[b]{2}{*}{ Area } & \multirow[b]{2}{*}{ Location } & \multirow[b]{2}{*}{ BA } & \multicolumn{3}{|c|}{ Peak Talairach coordinates } & \multirow[b]{2}{*}{$t$} & \multirow[b]{2}{*}{ CS } \\
\hline & & & $x$ & $y$ & $z$ & & & & & & $x$ & $y$ & $z$ & & \\
\hline \multirow[t]{2}{*}{ R Front. } & IFG & 44 & 48 & 12 & 19 & 6.02 & 124 & R Front. & IFG & 44 & 48 & 9 & 21 & 4.62 & 71 \\
\hline & MFG & 10 & 36 & 41 & 25 & 4.64 & 63 & & MFG & 6 & 27 & 10 & 47 & 6.31 & 283 \\
\hline \multirow[t]{7}{*}{ L/R Front. } & IFG & $6,8,44,9,46,32$ & -27 & 1 & 44 & 8.40 & 1668 & & & & & & & & \\
\hline & MFG & & & & & & & & & & & & & & \\
\hline & MedFG & & & & & & & & & & & & & & \\
\hline & PrecG & & & & & & & & & & & & & & \\
\hline & SFG & & & & & & & & & & & & & & \\
\hline & & & & & & & & LFront. & $\begin{array}{l}\text { IFG } \\
\text { MFG }\end{array}$ & $6,44,46$ & -27 & 4 & 49 & 6.06 & 1093 \\
\hline & & & & & & & & & PrecG & & & & & & \\
\hline R Par. & $\begin{array}{l}\text { IPL } \\
\text { SPL } \\
\text { Precun. }\end{array}$ & 7,40 & 27 & -58 & 46 & 9.34 & 1054 & R Par. & $\begin{array}{l}\text { IPL } \\
\text { Precun. }\end{array}$ & 40 & 42 & -40 & 43 & 6.11 & 1391 \\
\hline LPar. & $\begin{array}{l}\text { SPL } \\
\text { Precun. }\end{array}$ & $7,39,31$ & -27 & -67 & 23 & 10.19 & 1269 & LPar. & $\begin{array}{l}\text { SPL } \\
\text { Precun. }\end{array}$ & 7 & -30 & -54 & 43 & 6.25 & 1052 \\
\hline ROcc. & LG & 18 & 12 & -80 & -6 & 4.31 & 48 & R Occ. & LG & 17 & 12 & -88 & 4 & 4.53 & 1391 \\
\hline \multirow[t]{2}{*}{ L Occ. } & LG & 18,17 & -15 & -80 & -8 & 5.43 & 116 & L Occ. & LG & 18,17 & -18 & -73 & 0 & 3.94 & 1391 \\
\hline & Cuneus & & & & & & & & Cuneus & & & & & 4.71 & \\
\hline R Ins. & Insula & 13 & 33 & 22 & 2 & 4.75 & 44 & R Ins. & Insula & 13 & -39 & 25 & 2 & 4.24 & 38 \\
\hline L Ins. & Insula & 13 & -33 & 22 & 2 & 4.07 & 38 & L Basal & Striatum & & -21 & 5 & 13 & 5.02 & 1093 \\
\hline \multirow[t]{2}{*}{ R Cereb. } & & & 33 & -60 & -30 & 7.28 & 169 & R Cereb. & & & 39 & -63 & -30 & 5.51 & 133 \\
\hline & & & 18 & -77 & -11 & 4.31 & 48 & & & & & & & & \\
\hline L Cereb. & & & -30 & -60 & -30 & 3.99 & 23 & L Cereb. & & & -27 & -61 & -32 & 4.52 & 31 \\
\hline
\end{tabular}

AlphaSim cluster size $>22, p<0.001$.

R, Right; L, left; Front., frontal; Par., parietal; Occ., occipital, Ins., insular; Cereb., cerebellum; BA, Brodmann area; CS, cluster size; IFG, inferior frontal gyrus; MFG, middle frontal gyrus; MedFG, medial frontal gyrus; SFG, superior frontal gyrus; PrecG, precentral gyrus; IPL, inferior parietal lobule; SPL, superior parietal lobule; Precun., Precuneus; LG, lingual gyrus.

Table 3. Regions in which overlapping activations were observed between the training task [(dual 2-back) - (dual 0-back)] and the transfer task (dual WM updating baseline) in the active control group at pretest and at posttest

\begin{tabular}{|c|c|c|c|c|c|c|c|c|c|c|c|c|c|c|c|}
\hline \multicolumn{8}{|l|}{ Pretest } & \multicolumn{8}{|l|}{ Posttest } \\
\hline \multirow[b]{2}{*}{ Area } & \multirow[b]{2}{*}{ Location } & \multirow[b]{2}{*}{$B A$} & \multicolumn{3}{|c|}{ Peak Talairach coordinates } & \multirow[b]{2}{*}{$t$} & \multirow[b]{2}{*}{ CS } & \multirow[b]{2}{*}{ Area } & \multirow[b]{2}{*}{ Location } & \multirow[b]{2}{*}{ BA } & \multicolumn{3}{|c|}{ Peak Talairach coordinates } & \multirow[b]{2}{*}{$t$} & \multirow[b]{2}{*}{ CS } \\
\hline & & & $x$ & $y$ & $z$ & & & & & & $x$ & $y$ & $z$ & & \\
\hline R/L Front. & $\begin{array}{l}\text { IFG } \\
\text { MFG } \\
\text { MedFG }\end{array}$ & $6,9,45,46$ & -33 & 1 & 41 & 6.50 & 915 & & & & & & & & \\
\hline \multirow[t]{3}{*}{ R Front. } & IFG & 44 & 48 & 9 & 19 & 4.21 & 69 & R Front. & IFG & $6,8,9$ & 27 & 14 & 55 & 6.34 & 540 \\
\hline & MFG & 6,8 & 30 & 7 & 51 & 6.31 & 302 & & MFG & 10 & 42 & 41 & 19 & 5.12 & 148 \\
\hline & MFG & 10 & 45 & 44 & 21 & 4.55 & 78 & & $\begin{array}{l}\text { MFG } \\
\text { SFG }\end{array}$ & & & & & & \\
\hline \multirow[t]{2}{*}{ L Front. } & MFG & 10 & -39 & 52 & 16 & 4.25 & 29 & L Front. & $\begin{array}{l}\text { IFG } \\
\text { MFG } \\
\text { SFG }\end{array}$ & $6,9,44,46$ & -3 & 14 & 49 & 9.71 & 1323 \\
\hline & & & & & & & & & MFG & 10 & -30 & 49 & 11 & 5.13 & 67 \\
\hline R Par. & $\begin{array}{l}\text { Precuneus } \\
\text { IPL }\end{array}$ & 7,40 & 42 & -39 & 42 & 6.35 & 816 & R Par. & $\begin{array}{l}\text { Precuneus } \\
\text { IPL }\end{array}$ & 7,40 & 51 & -37 & 52 & 7.77 & 614 \\
\hline L Par. & $\begin{array}{l}\text { Precuneus } \\
\text { IPL }\end{array}$ & 7,40 & -39 & -48 & 36 & 7.24 & 862 & LPar. & $\begin{array}{l}\text { Precuneus } \\
\text { IPL }\end{array}$ & 7,40 & -45 & -46 & 49 & 8.19 & 755 \\
\hline ROCc. & MedOG & 19 & 33 & -76 & 10 & 4.56 & 816 & & & & & & & & \\
\hline L Occ. & MedOG & 19 & -33 & -76 & 12 & 4.45 & 862 & & & & & & & & \\
\hline \multirow[t]{3}{*}{ R Temp. } & MedTG & 39 & 36 & -70 & 17 & 4.56 & 816 & & & & & & & & \\
\hline & & & & & & & & L Temp. & STG & 22 & -48 & 11 & -1 & 3.69 & 1323 \\
\hline & & & & & & & & L Ins. & Insula & 13 & -30 & 25 & 2 & 5.13 & 173 \\
\hline L Basal & Striatum & & -15 & 3 & 16 & 4.84 & 915 & L Basal & Striatum & & -18 & 6 & 14 & 5.28 & 173 \\
\hline L Cereb. & & & -39 & -63 & -30 & 4.45 & 862 & L Cereb. & & & -39 & -70 & -29 & 4.08 & 22 \\
\hline
\end{tabular}


Table 4. Regions in which overlapping activations were observed between the training task [(dual 2-back) - (dual 0-back)] and the transfer task (dual WM updating baseline) in the passive control group at pretest and at posttest

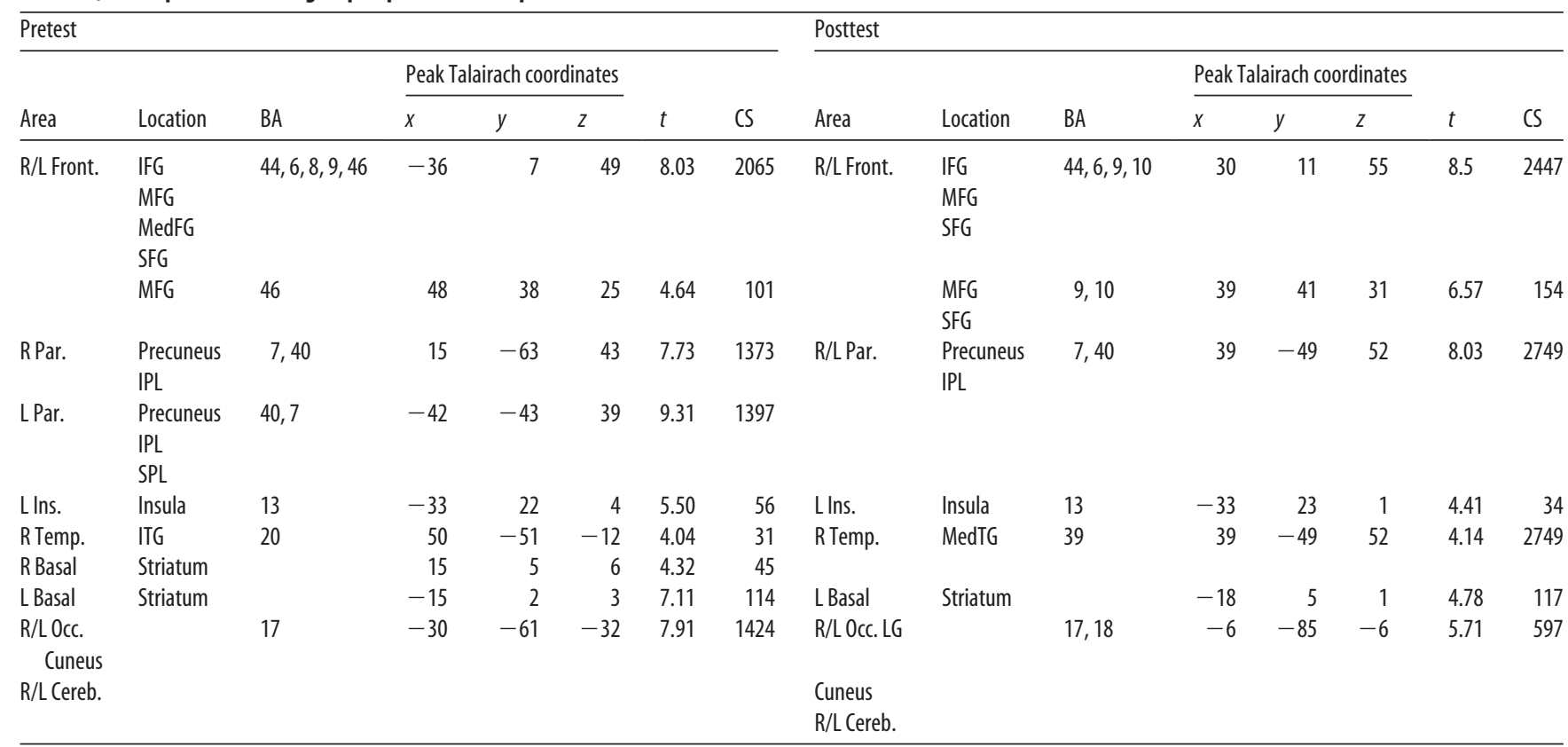

AlphaSim cluster size $>22, p<0.001$.

R, Right; L, left; Front., frontal; Par., parietal; Occ., occipital, Temp., temporal; Ins., insular; Cereb., cerebellum; BA, Brodmann area; CS, cluster size; IFG, inferior frontal gyrus; MFG, middle frontal gyrus; MedFG, medial frontal gyrus; SFG, superior frontal gyrus; IPL, inferior parietal lobule; SPL, superior parietal lobule; LG, lingual gyrus; ITG, inferior temporal gyrus; MedTG, medial temporal gyrus.

updating task situations, although they showed overlap in the frontoparietal networks. This confirms that overlap of the frontoparietal network at pretest or a certain pattern of training related decrease of that overlap cannot be a decisive precondition for observing transfer (see also Dahlin et al., 2008).

\section{Training-related activation changes}

Next, we analyzed the particular training-related changes that occur in the pattern of fMRI activations in the training and transfer task separately. By analyzing these changes, we aimed to approach our second hypothesis that specific training-related activation increases in the striatum are specifically associated with transfer after dual $n$-back training to the dual WM updating task.

\section{Dual $\boldsymbol{n}$-back task}

Training group

Analyses of the contrast [(dual 2-back pretest - dual 0-back pretest) - (dual 2-back posttest - dual 0-back posttest)] showed decreases in extended regions of the frontoparietal network, including the bilateral premotor cortex, the bilateral PFC, and the right inferior parietal lobule (Fig. 5, Table 5). In addition, we observed a decrease of activation from pretest to posttest in the right anterior cingulate cortex and in the left posterior cingulate cortex, but an activation increase in the superior temporal lobe. With a more lenient threshold of $p<0.05$ (uncorrected; keeping the cluster size of $>22$ voxels) we also observed an activation increase in the left and right striatum accompanied by an activation decrease in the bilateral thalamus.

\section{Active control group}

In the active control group, we found a decrease of activation from pretest to posttest only in the right middle frontal gyrus and in the left inferior parietal lobule (Fig. 5, Table 5). We did not observe any increases of activation.
Passive control group

In the passive control group, we found no activation decreases or increases from pretest to posttest at all (Fig. 5).

\section{Dual WM updating task}

Training group

As can be seen in Figure 6 (Table 5), in the training group, we observed strong activation increases from pretest to posttest in the bilateral occipital cortex as well as bilaterally in the striatum.

\section{Active control group}

In the active control group, there were no significant decreases or increases observed (Fig. 6).

\section{Passive control group}

In the passive control group, there were no significant decreases or increases observed (Fig. 6).

Together, the training group showed large decreases in the frontoparietal network in the training task, accompanied by an increase of activation in the striatum in both the training and the transfer WM updating tasks. In addition, the active control group showed decreased activation in the frontoparietal network, but to a much lesser extent than the training group, which reflects the observation of some improvement on a behavioral level. There were no activation changes in the transfer task in either of the control groups.

Most importantly, the apparent differences in the activation changes between the three groups in the dual WM updating task were confirmed by a subsequent whole-brain groupwise comparison. That is, for the dual WM updating transfer task, a flexible factorial ANOVA with the factors group (training vs active control vs passive control) and session (pretest vs posttest) revealed significant differences in the pretest to posttest activation changes in the right striatum between the three groups (AlphaSim cluster size $>22, p<0.001$ ) and with a more lenient threshold of $p<$ 


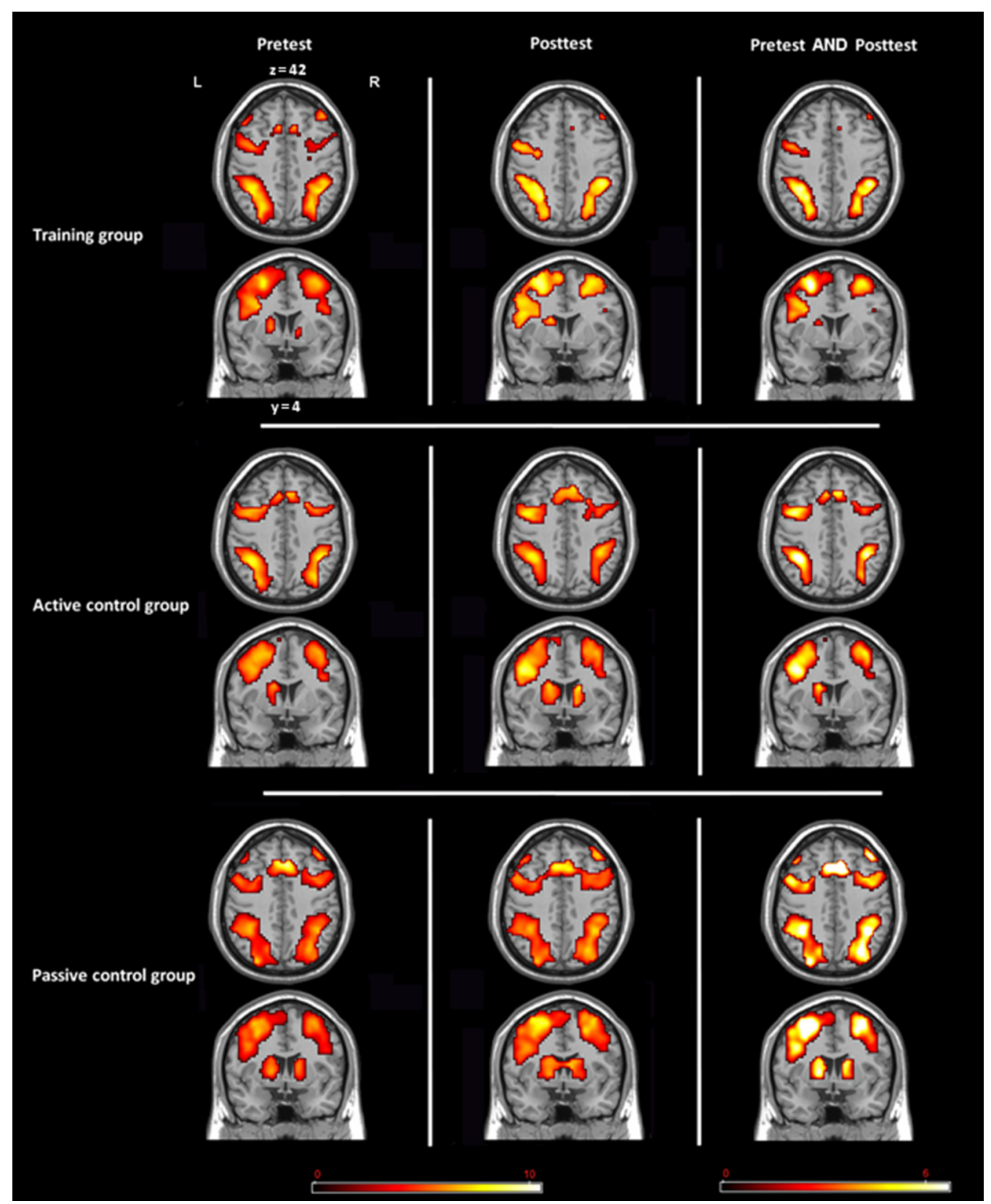

Figure 3. Regions in which overlapping activations were observed between the training (dual 2-back) and the transfer (dual WM updating) tasks in the training (top), active control (center), and passive control (bottom) groups. Overlap is depicted separately for pretest (left) and posttest (center), along with a conjunction of overlapping activations at pretest and at posttest (right) (see main text) (AlphaSim cluster size $>22, p<0.001$ ).

0.05 (uncorrected; keeping the cluster size of $>22$ voxels) in the bilateral striatum and in the bilateral cuneus. To visualize the differences in the activation changes between the three groups in the dual WM updating task, we extracted percentage signal changes (PSCs) from the activated clusters of interest using Mars$\mathrm{BaR}$ (http://marsbar.sourceforge.net/; Brett et al., 2002). Clusters of interest were defined at local peak activity maxima of the training group's activity change in the striatum (posttest pretest) from the whole-brain analyses for the dual WM updating task with AlphaSim correction cluster size $>22, p<$ 0.05 . This produced clusters of interest in the right striatum at $x=6, y=17, z=-2$ and in the left striatum at $x=-15, y=$ $17, z=-11$. To exclude the possibility that the other groups showed a similar activation increase in a slightly different location, we masked the activation cluster with a sphere $6 \mathrm{~mm}$ in diameter and extracted the mean PSC from that region of interest (ROI) over a time window of $4-25 \mathrm{~s}$ after sequence onset (because sequences had different lengths, this time window was set according to the shortest sequence to ensure that, for each sequence, only the updating and not the response phase was modeled). As can be seen in Figure 7, we observed an increase of the PSC in the striatum for the training group, whereas the PSC in the two other groups decreased from pretest to posttest.

From the same clusters, we then extracted the PSCs of the dual $n$-back task to investigate group- and task-specific striatal activation changes from pretest to posttest. A group (training vs active control vs passive control) $\times$ session (pretest vs posttest) $\times$ task (dual $n$-back vs dual WM updating) ANOVA revealed a significant group $\times$ session interaction both in the left striatum cluster 


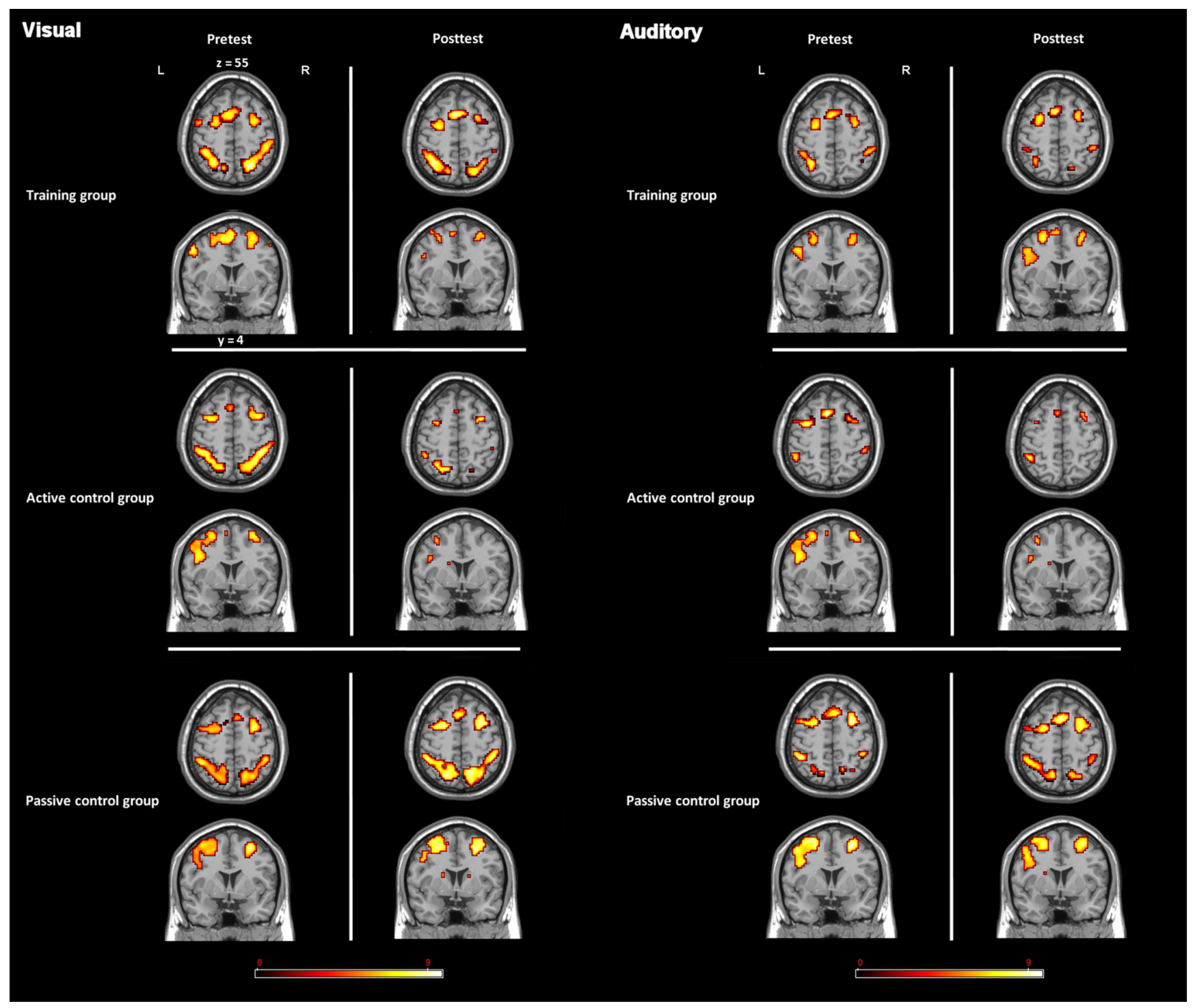

Figure 4. Regions in which overlapping activations were observed between the single versions of the tasks (single 2-back and single WM updating) in the training (top), active control (center), and passive control (bottom) groups. Overlap is depicted separately for pretest (left) and posttest (right). Visual tasks are presented on the left and auditory tasks on the right.

$\left(F_{(2,51)}=5.84, p<0.05, \eta_{p}^{2}=0.19\right)$ and in the right striatum cluster $\left(F_{(2,51)}=4.65, p<0.05, \eta_{p}^{2}=0.15\right)$, whereas the group $\times$ session $X$ task interaction was in both clusters not significant $(p>0.33)$. That is, the training group's striatal activation increase was significantly larger than those of the control group and this effect was similar in the dual $n$-back and the dual WM updating task.

For the training task, a whole-brain comparison between the training group and the passive control group showed significant differences (AlphaSim cluster size $>22, p<0.05$ ) in the amount of activation changes in the striatum from pretest to posttest, whereas the corresponding differences with the active control group did not survive the chosen significance threshold.

All in all, these analyses confirm significant group-specific differences in the striatum, with an increase of the PSC in the training group, but no significant increases in the active and the passive control group.
Relationship between activation increase in the striatum and training-related WM performance

Subsequently, we investigated whether the observed training and transfer effects in the WM tasks are related to the activation increases in the striatum. For that purpose, we analyzed differences in the striatal activation changes between the highest- and lowest-gaining participants in the training group separately for the dual $n$-back and the dual WM updating task. First, we divided the participants in the training group into low-gaining $(n=9)$ and high-gaining $(n=9)$ groups by using a median split of their gain in the dual $n$-back task. Then, we extracted the PSC from ROIs with a similar procedure as described above for the dual WM updating task: clusters of interest were defined at local peak activity maxima of the training group's activity change in the striatum (posttest pretest) from the whole-brain analyses for the dual $n$-back task with AlphaSim correction cluster size $>22, p<0.05$. This analysis yielded clusters of interest (masked by a sphere $6 \mathrm{~mm}$ 


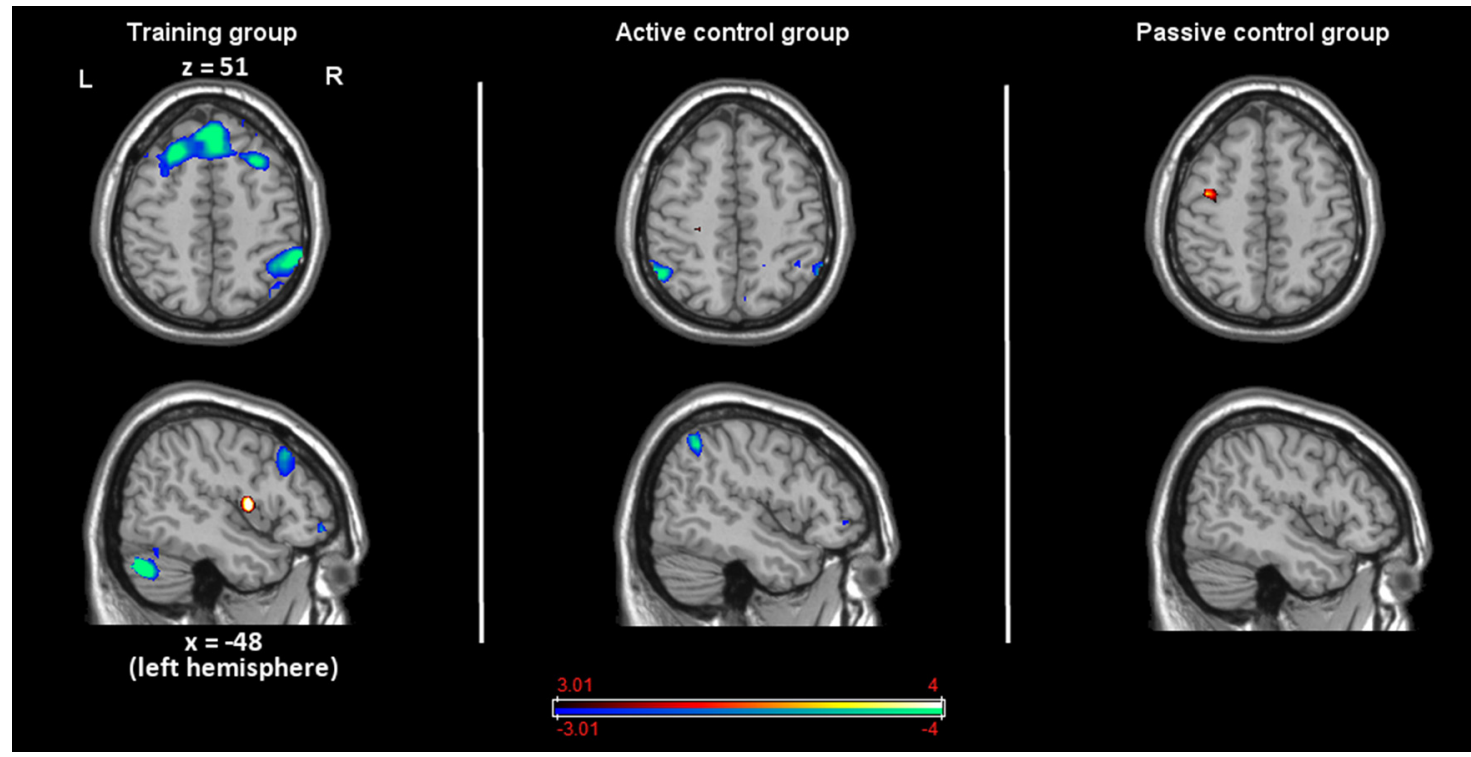

Figure 5. Activation decreases and increases in the dual $n$-back task in the training group (left), the active control group (middle), and the passive control group (right). Blue indicates decreases and red increases (AlphaSim cluster size $>22, p<0.001$ ).

Table 5. Increases and decreases after intervention in the training and transfer tasks

\begin{tabular}{|c|c|c|c|c|c|c|c|c|c|}
\hline & \multirow[b]{2}{*}{ Side } & \multirow[b]{2}{*}{ Area } & \multirow[b]{2}{*}{ Location } & \multirow[b]{2}{*}{ BA } & \multicolumn{3}{|c|}{ Peak Talairach coordinates } & \multirow[b]{2}{*}{$t$} & \multirow[b]{2}{*}{ CS } \\
\hline & & & & & $x$ & $y$ & $z$ & & \\
\hline \multicolumn{10}{|c|}{ Training dual $n$-back task } \\
\hline \multicolumn{10}{|c|}{ Training group } \\
\hline \multirow[t]{10}{*}{ Decreases } & $\mathrm{R}$ & Frontal & Inferior frontal gyrus & 13 & 33 & 13 & -14 & 5.33 & 26 \\
\hline & & & Middle frontal gyrus & 8 & 33 & 18 & 39 & 4.62 & 28 \\
\hline & & & Superior frontal gyrus & 10 & 24 & 55 & 24 & 4.49 & 27 \\
\hline & L & Frontal & Medial frontal gyrus & 8 & -12 & 30 & 37 & 4.8 & 269 \\
\hline & & & Superior frontal gyrus & 10 & -21 & 57 & 4 & 5.89 & 110 \\
\hline & $\mathrm{R}$ & Parietal & Inferior parietal lobule & 40 & 56 & -42 & 41 & 4.87 & 125 \\
\hline & $\mathrm{R}$ & Limbic & Anterior cingulate gyrus & 24 & 3 & 3 & 24 & 5.61 & 47 \\
\hline & L & Limbic & Posterior cingulate gyrus & 31 & -3 & -40 & 31 & 4.74 & 52 \\
\hline & $\mathrm{R}$ & Cerebellum & & & 48 & -72 & -35 & 5.37 & 205 \\
\hline & $\mathrm{L}$ & Cerebellum & & & -45 & -75 & -33 & 6.65 & 126 \\
\hline Increases & $\mathrm{L}$ & Frontal & Precentral gyrus & 6 & -50 & -1 & 6 & 8.3 & 73 \\
\hline \multicolumn{10}{|c|}{ Active control group } \\
\hline \multirow[t]{3}{*}{ Decreases } & $\mathrm{R}$ & Frontal & Middle frontal gyrus & 10 & 39 & 51 & -4 & 5.28 & 38 \\
\hline & $\mathrm{L}$ & Frontal & Inferior frontal gyrus & 10 & -36 & 44 & -2 & 5.26 & 36 \\
\hline & L & Parietal & Inferior parietal lobule & 40 & -50 & -54 & 36 & 4.37 & 30 \\
\hline \multicolumn{10}{|c|}{$\begin{array}{l}\text { Transfer dual WM updating task } \\
\text { Training group }\end{array}$} \\
\hline \multirow[t]{3}{*}{ Increases } & $\mathrm{R} / \mathrm{L}$ & Occipital & Calcarine gyrus, cuneus & 19,23 & 18 & -64 & 7 & 6.54 & 502 \\
\hline & $\mathrm{R}$ & Basal & Striatum & & 21 & 14 & 2 & 5.01 & 28 \\
\hline & L & Basal & Striatum & & -15 & 5 & 4 & 4.42 & 46 \\
\hline
\end{tabular}

AlphaSim correction $p<0.001$, cluster size $>22$.

R, Right; L, left; BA, Brodmann area; CS, cluster size. Note. There were no training-related decreases in the training group in the transfer dual WM updating task. In the active control group, there were no training-related increases observed in the dual $n$-back task and no activation changes in the transfer dual WM updating task. There were no training-related activation changes observed in the passive control group in either tasks.

in diameter) in the right striatum at $x=12, y=17, z=-11$ and in the left striatum at $x=-15, y=8, z=-11$. In the dual $n$-back task, we extracted the mean PSC over a time window of 4-34 s after stimulus onset. To compare the change of activation with behavior, we collapsed the extracted PSCs of the ROIs on the right and the left side. A one-way ANOVA on the striatal activation change between the two groups yielded a significant result $\left(F_{(1,16)}=4.82, p<0.05\right.$, Cohen's $\left.d=1.04\right)$. That is, the participants in the high-gaining group exhibited a larger striatal activation increase from pretest to posttest than the low-gaining group (Fig. 8, left). A similar analysis was conducted for the transfer dual WM updating task. We divided the participants in the training group into low-gaining $(n=9)$ and high-gaining $(n=9)$ groups by using a median split of their gain in the dual WM updating task. The PSC in the striatum was extracted for the dual WM updating task as defined above. This analysis did not reach significance $(p>0.77)$.

In conclusion, these analyses show an association between the observed changes in behavior and activation changes in the striatum, at least in the dual $n$-back task.

Dual-task specific activation changes

To determine whether the observed behavioral and neural changes are due to improvements solely in dual-task coordination skills rather than in WM, we analyzed dual-task-related 


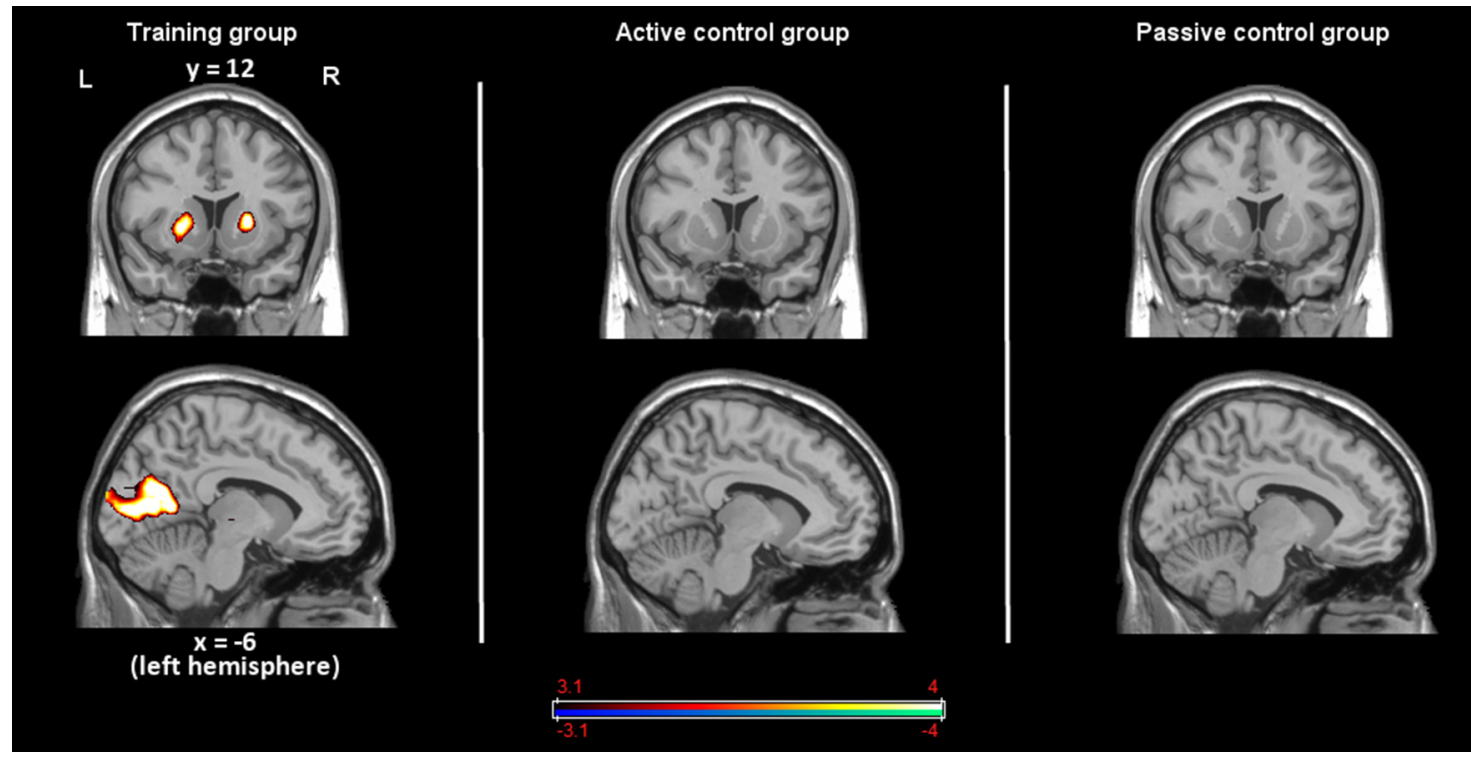

Figure 6. Activation decreases and increases in the transfer WM updating task in the training group (left), the active control group (middle), and the passive control group (right). Blue indicates decreases and red increases (AlphaSim cluster size $>22, p<0.001$ ).
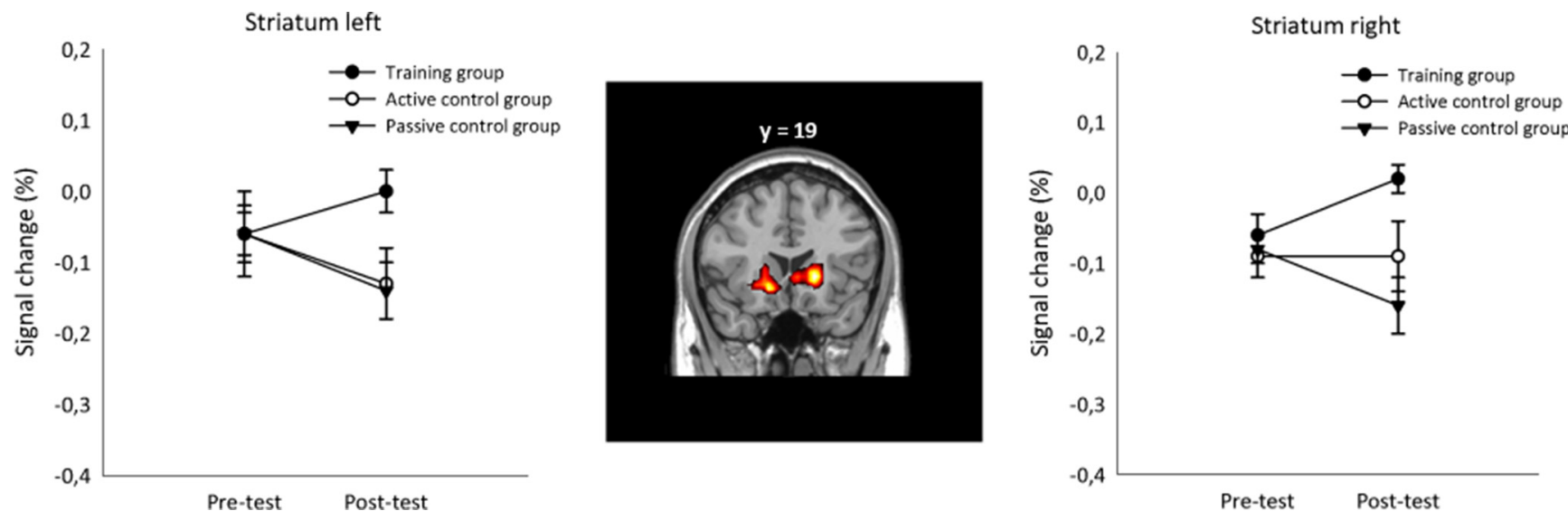

Figure 7. Percentage signal change values extracted from the striatum to visualize activation change in the dual WM updating task from pretest to posttest in each group (percentage signal change extracted from left striatum at $x=-15, y=17, z=-11$; right striatum at $x=6, y=17, z=-2$ ).

activation changes with a dual-task coordination contrast [dual 2-back - (AV single 2-back + VS single 2-back)] (see also Schubert and Szameitat, 2003; Szameitat et al., 2011). With this contrast, we extracted neural activation related exclusively to dual-task coordination. The contrast was first calculated in each group for pretest and then for posttest, after which we calculated a contrast for decreased activation from pretest to posttest [(dual-task contrast pretest - dual-task contrast posttest)] and a contrast for increased activation from pretest to posttest [(dual-task contrast posttest - dualtask contrast pretest)].

The training group showed an activation decrease (AlphaSim cluster size $>22, p<0.001$ ) from pretest to posttest left-sided in the PFC (middle frontal gyrus, BA 8; superior frontal gyrus, BAs 8/10; inferior frontal gyrus, BA 45) and the occipital cortex (precuneus, BA 19) (Table 6). This pattern of activations corresponds to dual-task coordination activations reported in previous studies (Szameitat et al., 2002; Schubert and Szameitat, 2003; Stelzel et al., 2008). A training-related decrease in these regions thus implies that the demand for dual-task coordination was reduced at posttest. However, a similar pattern of activation decreases was observed in both the active and the passive control group (Table 6). Comparisons of the activation changes between the training group and the active control group, as well as between the training group and the passive control group [i.e., a flexible factorial ANOVA with the factors group (training vs active control and training vs passive control) and session (pretest vs posttest)], did not yield significant differences (AlphaSim cluster size $>22, p<$ $0.001)$. Because the dual-task analyses revealed that all groups showed activation decreases in dual-task-related regions and because we found no differences in the activation changes between the groups, we can exclude the possibility that the observed behavioral improvements in the training group were due solely to improved dual-task coordination skills as a result of dual $n$-back training. It should be noted that the contrasts that we calculated for dual-task specific activations included only the 2-back version of the tasks, that is, without assessing a change in WM load. This is important because the activation changes that we reported earlier specifically for the dual 
$n$-back task took into account the changing WM load because the contrast subtracted dual 0-back from the dual 2-back task. Therefore, the striatal activation decrease that we found in the dual-task coordination analyses in all groups (Table 6) should be distinguished from the striatal activation increase that we observed exclusively in the training group for the task analyses above.

\section{Discussion}

We investigated whether dual $n$-back training improves general cognitive resources or a task-specific process. After training, the training group outperformed both control groups in the training task and they were the only group to show transfer to a dualmodality WM updating task. The training group did not perform better than the active control group in single versions of the training task nor were there differences between any of the groups in their improvement in single versions of the WM updating transfer task. We found in the training group reduced overlapping activations of the training and transfer task. We additionally observed a dual $n$-back trainingrelated decrease in the frontoparietal network in the training task and an increase in the striatum in the training and the transfer task. The control groups showed only little (active control group) or no change (passive control group) in the activation patterns from pretest to posttest.

These results support our second hypothesis: dual $n$-back training did not improve general cognitive resources, but rather a specific cognitive process of WM updating. This conclusion is based on the findings that frontoparietal activation overlap was not associated with transfer, that the amount of activation increased in the striatum only in the training group, and that transfer effects were tied to highly similar task situations. Had general cognitive resources improved, we should have observed more widespread transfer effects (i.e., transfer to the single-task versions of the training and the WM updating task) and overlap in the tasks' frontoparietal activations that would be a prerequisite for transfer.

The present results are especially interesting in the light of the findings of Dahlin et al. (2008), who showed that striatal activation mediates transfer from WM updating training to an $n$-back task. With the present results we bring important new insight and refinement into the prerequisites of transfer. First, due to its dual-task coordination component, the dual $n$-back training paradigm presumably engages the PFC stronger than the single-task used in Dahlin et al. (2008). Consequently, the dual $n$-back might be regarded as a more appropriate training task than a single- task for testing whether a general capacity boost underlies transfer effects. Second, in Dahlin et al. (2008), the transfer tasks included a task that clearly requires updating processes that are engaged in their training task (the $n$-back task) and a task requiring inhibition of prepotent responses, and thus processes that are not involved in their training task (Stroop task). In the current study, updating processes played a central role in all tasks, but transfer was observed only to the dual WM updating task. These results demonstrate that transfer requires the sharing of very similar processes (in the present study, the updating of stimuli from two different modalities). We emphasize that the dual $n$-back task and the dual WM updating task differed with regard to stimuli and task rules, but transfer occurred only when the training and the transfer task shared a similar cognitive process, that is, the updating of two memory lists.

In contrast to single $n$-back training, dual $n$-back training seems demanding enough to fulfill the requirements for improving updating processes in a way that profits performance in the dual WM updating task. This implies improved coordination of stimuli from two different modalities, which is not necessary when only one modality is processed.

Studies have shown that it is possible to train executive control processes of dual-task coordination (Liepelt et al., 2011; Strobach et al., 2012). Such training effects are observed when subjects train two tasks simultaneously compared with training of the single subtasks and the improvements transfer to untrained dual tasks. The inclusion of an active control group with single $n$-back training allowed us to identify training-related changes and transfer effects that are related to the executive control components of task coordination. Specific analyses on dual-task coordination in the training task revealed that all groups showed equivalent activation decreases in dual-task specific brain regions. These analyses confirmed that the observed improvements in the training group cannot be attributed to sole improvements in dual-task coordination skills.

Updating is regarded as a core executive function (Miyake et al., 2000). The improvement in updating could indicate improvements in some general domain (in contrast to a very specific process improvement). However, the current findings suggest that this was not the case. Had more general resources profited from training, one should expect transfer also to the single versions of the tasks used in the current study. Such effects were not found. From this perspective, it also seems that a very specific process was improved. Regarding studies that have shown dual $n$-back training-related improvements in fluid intelligence (Jaeggi et al., 2008, 2010), we suggest that if the trained updating processes are crucial in the intelligence test, then transfer to such a test is possible. Indeed, Jaeggi et al. (2010) observed strong 
Table 6. Training-related increases and decreases in the dual $n$-back task from the dual-task contrast [dual 2-back - (visual single 2-back + auditory single 2-back)]

\begin{tabular}{|c|c|c|c|c|c|c|c|c|c|}
\hline & \multirow[b]{2}{*}{ Side } & \multirow[b]{2}{*}{ Area } & \multirow[b]{2}{*}{ Location } & \multirow[b]{2}{*}{ BA } & \multicolumn{3}{|c|}{ Talairach coordinates } & \multirow[b]{2}{*}{$t$} & \multirow[b]{2}{*}{ CS } \\
\hline & & & & & $x$ & $y$ & $z$ & & \\
\hline \multicolumn{10}{|l|}{ Training group } \\
\hline \multirow[t]{4}{*}{ Decreases } & L & Frontal & Inferior frontal gyrus & 45 & -53 & 26 & 17 & 4.22 & 40 \\
\hline & & & Superior frontal gyrus & $8 /$ & -18 & 39 & 45 & 6.34 & 165 \\
\hline & & & & 10 & -12 & 64 & 14 & 6.25 & 49 \\
\hline & L & Parietal & Precuneus & 19 & -33 & -66 & 43 & 6.09 & 256 \\
\hline \multirow[t]{2}{*}{ Increases } & $\mathrm{R}$ & Frontal & Inferior frontal gyrus & 44 & 56 & 17 & 12 & 5.12 & 86 \\
\hline & $\mathrm{R}$ & Parietal & Inferior parietal lobule & 40 & 65 & -43 & 24 & 5.46 & 68 \\
\hline \multicolumn{10}{|c|}{ Active control group } \\
\hline \multirow[t]{3}{*}{ Decreases } & L & Frontal & Middle frontal gyrus & 8 & -42 & 10 & 44 & 5.45 & 38 \\
\hline & & & Medial frontal gyrus & 10 & -15 & 31 & -8 & 6.49 & 144 \\
\hline & L & Parietal & Precuneus & 19 & -27 & -77 & -37 & 8.00 & 29 \\
\hline Increases & $\mathrm{R}$ & Temporal & Middle temporal gyrus & 37 & 59 & -62 & 5 & 5.05 & 34 \\
\hline \multicolumn{10}{|c|}{ Passive control group } \\
\hline \multirow[t]{7}{*}{ Decreases } & L & Frontal & Inferior frontal gyrus & 13 & -45 & 26 & 10 & 4.84 & 78 \\
\hline & & & Precentral gyrus & $4 /$ & -36 & -22 & 52 & 5.88 & 71 \\
\hline & & & & 44 & -50 & 11 & 6 & 4.09 & 36 \\
\hline & & & Middle frontal gyrus & 6 & -24 & 7 & 46 & 5.24 & 123 \\
\hline & L & Temporal & Middle temporal gyrus & 39 & -48 & -73 & 17 & 5.25 & 58 \\
\hline & $\mathrm{L}$ & Occipital & Inferior occipital gyrus & 18 & -36 & -94 & -6 & 7.51 & 43 \\
\hline & L & Basal & Striatum & & -15 & 25 & -5 & 5.13 & 67 \\
\hline \multirow[t]{3}{*}{ Increases } & $\mathrm{R}$ & Frontal & Middle frontal gyrus & 46 & 45 & 38 & 25 & 4.34 & 32 \\
\hline & & & Superior frontal gyrus & 10 & 24 & 57 & 6 & 6.10 & 56 \\
\hline & $\mathrm{R}$ & Temporal & Middle temporal gyrus & 39 & 48 & -72 & 25 & 8.00 & 932 \\
\hline
\end{tabular}

AlphaSim correction $p<0.001$, cluster size $>22$.

$R$, Right; L, left; BA, Brodmann area.

correlations between $n$-back performance and performance in intelligence tests ( Raven, 1990; Hossiep et al., 1999). According to the investigators, this correlation can explain the observed transfer effect from dual $n$-back training to fluid intelligence in their previous study (Jaeggi et al., 2008, 2010). Friedman et al. (2006) showed correlations between updating performance and fluid intelligence. They concluded that tests such as the Raven's Advanced Progressive Matrices may measure updating skills. It should be noted that, because several other studies have not replicated the dual $n$-back training-related improvement in fluid intelligence, the possibility to increase intelligence with dual $n$-back training remains a matter of debate (Chooi and Thompson, 2012; Salminen et al., 2012; Redick et al., 2013; Thompson et al., 2013).

An issue that deserves consideration is why single $n$-back training in our study did not produce transfer, whereas single updating training in Dahlin et al. (2008) transferred to $n$-back performance. In our view, this difference is related to the different WM requirements involved in the training tasks. As proposed by several investigators (e.g., Jaeggi et al., 2010), the $n$-back task is based more on less demanding recognition processes, whereas the WM updating task requires the coordination of recalled items during WM updating. In addition, in the present study, the training and transfer tasks included a dual-task component, whereas in the study by Dahlin et al. (2008), both tasks were single visual WM tasks. Finally, whereas in Dahlin et al. (2008), the transfer effects were compared against a passive control group, in the present study, we included an active and a passive control group. Comparisons against an active control group allowed us to control for the occurrence of unspecific effects of training that could complicate the interpretation of transfer effects (Shipstead et al., 2012).

The observed pattern of neural activations is consistent with previous findings on activation changes after WM training (Hempel et al., 2004; Klingberg, 2010, Kühn et al., 2013, Schneiders et al., 2012; Buschkuehl et al., 2014). The PFC activation decrease in the training group implies less requirement for cognitive and attentional control in the dual $n$-back task after training than before training. Activation focus shifted to a more process-specific region: activation increased in the striatum in the training and the transfer task. Improved WM updating and increased striatal activation is consistent with theories that describe striatum's role in WM updating (Frank et al., 2001; Gruber et al., 2006; Baier et al., 2010; Voytek and Knight, 2010). The striatum is required in regulating which information enters WM (Alexander et al., 1986; McNab and Klingberg, 2008). Dahlin et al. (2008) proposed that updating during training induced changes in the striatal dopaminergic system (see also Bäckman et al., 2011), which underlies WM updating processes by modulating related neural activations in the PFC (Cohen et al., 2002; O'Reilly, 2006).

Comparing high and low gainers revealed associations between behavioral improvements and increased striatal activation. Although these results should be interpreted with caution (we did not find a correlation between behavioral and neuronal data), our analyses imply a manifestation of striatal activation increase in WM updating improvement.

Interestingly, a lenient threshold of $p<0.05$ revealed a noteworthy activation pattern in the thalamus. In the training group, we observed decreased thalamus activation in the training task, 
but no change in the transfer task, whereas both control groups showed increased thalamus activation in the transfer task. Regarding learning models on the PFC and basal ganglia (O'Reilly and Frank, 2006), this finding could reflect thalamus participation in training and transfer effects. This possibility would be interesting to pursue in future studies.

In conclusion, we showed that dual $n$-back training improved a specific cognitive process, WM updating, and the trainingrelated changes were observed on a behavioral and a neuronal level. The results provide further evidence that overlapping frontoparietal activations alone do not produce transfer; instead, in training circumstances as in the present study, transfer occurs when neuronal activation increases in brain regions that are specifically associated with the transferred process. This implies that the training-related optimization of specific skills and their implementation in the transfer task are associated with the improvement in the transfer task.

\section{References}

Alexander GE, DeLong MR, Strick PL (1986) Parallel organization of functionally segregated circuits linking basal ganglia and cortex. Annu Rev Neurosci 9:357-381. CrossRef Medline

Au J, Sheehan E, Tsai N, Duncan GJ, Buschkuehl M, Jaeggi SM (2015) Improving fluid intelligence with training on working memory: a metaanalysis. Psychon Bull Rev 22:366-377. CrossRef Medline

Bäckman L, Nyberg L, Soveri A, Johansson J, Andersson M, Dahlin E, Neely AS, Virta J, Laine M, Rinne JO (2011) Effects of working-memory training on striatal dopamine release. Science 333:718. CrossRef Medline

Baddeley A, Della Sala S (1996) Working memory and executive control. Philos Trans R Soc B Biol Sci 351:1397-1404. CrossRef

Baier B, Karnath HO, Dieterich M, Birklein F, Heinze C, Müller NG (2010) Keeping memory clear and stable: the contribution of human basal ganglia and prefrontal cortex to working memory. J Neurosci 30:9788-9792. CrossRef Medline

Brett M, Anton JL, Valabregue R, Poline JB (2002) Region of interest analysis using an SPM toolbox [abstract]. Presented at the 8th International Conference on Functional Mapping of the Human Brain, Sendai, Japan, June 2-6. Available on CD-ROM in NeuroImage, Vol 16, No 2.

Buschkuehl M, Jaeggi SM, Kobel A, Perrig WJ (2007) BrainTwisterAufgabensammlung für kognitives Training, Version 1.0.1. Manual und CD. Institut für Psychologie, Universität Bern.

Buschkuehl M, Hernandez-Garcia L, Jaeggi SM, Bernard JA, Jonides J (2014) Neural effects of short-term training on working memory. Cogn Affect Behav Neurosci 14:147-160. CrossRef Medline

Chooi W-T, Thompson LA (2012) Working memory training does not improve intelligence in healthy young adults. Intelligence 40:531-542. CrossRef

Cohen JD, Braver TS, Brown JW (2002) Computational perspectives on dopamine function in prefrontal cortex. Curr Opin Neurobiol 12:223229. CrossRef Medline

Dahlin E, Neely AS, Larsson A, Bäckman L, Nyberg L (2008) Transfer of learning after updating training mediated by the striatum. Science 320 : 1510-1512. CrossRef Medline

Frank MJ, Loughry B, O’Reilly RC (2001) Interactions between frontal cortex and basal ganglia in working memory: a computational model. Cogn Affect Behav Neurosci 1:137-160. CrossRef Medline

Friedman NP, Miyake A, Corley RP, Young SE, Defries JC, Hewitt JK (2006) Not all executive functions are related to intelligence. Psychol Sci 17:172179. CrossRef Medline

Gruber AJ, Dayan P, Gutkin BS, Solla SA (2006) Dopamine modulation in the basal ganglia locks the gate to working memory. J Comput Neurosci 20:153-166. CrossRef Medline

Hempel A, Giesel FL, Garcia Caraballo NM, Amann M, Meyer H, Wüstenberg T, Essig M, Schröder J (2004) Plasticity of cortical activation related to working memory during training. Am J Psychiatry 161:745-747. CrossRef Medline

Hossiep R, Turck D, Hasella M (1999) Bochumer Matrizentest: BOMAT: advanced short version. Göttingen: Hogrefe.

Jaeggi SM, Buschkuehl M, Jonides J, Perrig WJ (2008) Improving fluid in- telligence with training on working memory. Proc Natl Acad Sci U S A 105:6829-6833. CrossRef Medline

Jaeggi SM, Buschkuehl M, Perrig WJ, Meier B (2010) The concurrent validity of the N-back task as a working memory measure. Memory 18:394412. CrossRef Medline

Jonides J (2004) How does practice makes perfect? Nat Neurosci 7:10-11. CrossRef Medline

Karbach J, Kray J (2009) How useful is executive control training? Age differences in near and far transfer of task-switching training. Dev Sci 12: 978-990. CrossRef Medline

Karbach J, Verhaeghen P (2014) Making working memory work: a metaanalysis of executive-control and working memory training in older adults. Psychol Sci 25:2027-2037. CrossRef Medline

Klingberg T (2006) Development of a superior frontal-intraparietal network for visuo-spatial working memory. Neuropsychologia 44:21712177. CrossRef Medline

Klingberg T (2010) Training and plasticity of working memory. Trends Cogn Sci 14:317-324. CrossRef Medline

Kühn S, Schmiedek F, Noack H, Wenger E, Bodammer NC, Lindenberger U, Lövden M (2013) The dynamics of change in striatal activity following updating training. Hum Brain Mapp 34:1530-1541. CrossRef Medline

Liepelt R, Strobach T, Frensch P, Schubert T (2011) Improved intertask coordination after extensive dual-task practice. Q J Exp Psychol (Hove) 64:1251-1272. CrossRef Medline

Lilienthal L, Tamez E, Shelton JT, Myerson J, Hale S (2013) Dual $n$-back training increases the capacity of the focus of attention. Psychon Bull Rev 20:135-141. CrossRef Medline

Lustig C, Shah P, Seidler R, Reuter-Lorenz PA (2009) Aging, training, and the brain: a review and future directions. Neuropsychol Rev 19:504-522. CrossRef Medline

McNab F, Klingberg T (2008) Prefrontal cortex and basal ganglia control access to working memory. Nat Neurosci 11:103-107. CrossRef Medline

Miller EK, Cohen JD (2001) An integrative theory of prefrontal cortex function. Annu Rev Neurosci 24:167-202. CrossRef Medline

Miyake A, Friedman NP, Emerson MJ, Witzki AH, Howerter A, Wager TD (2000) The unity and diversity of executive functions and their contributions to complex "frontal lobe" tasks: a latent variable analysis. Cogn Psychol 41:49-100. CrossRef Medline

Nichols T, Brett M, Andersson J, Wager T, Poline JB (2005) Valid conjunction inference with the minimum statistic. Neuroimage 25:653-660. CrossRef Medline

O’Reilly RC (2006) Biologically based computational models of high-level cognition. Science 314:91-94. CrossRef Medline

O’Reilly RC, Frank MJ (2006) Making working memory work: A computational model of learning in the prefrontal cortex and basal ganglia. Neural Comput 18:283-328. CrossRef Medline

Raven JC (1990) Advanced progressive matrices: sets I, II. Oxford: OUP.

Redick TS, Shipstead Z, Harrison TL, Hicks KL, Fried DE, Hambrick DZ, Kane MJ, Engle RW (2013) No evidence of intelligence improvement after working memory training: a randomized, placebo-controlled study. J Exp Psychol Gen 142:359-379. CrossRef Medline

Salminen T, Strobach T, Schubert T (2012) On the impacts of working memory training on executive functioning. Front Hum Neurosci 6:166. CrossRef Medline

Schneiders JA, Opitz B, Tang H, Deng Y, Xie C, Li H, Mecklinger A (2012) The impact of auditory working memory training on the fronto-parietal working memory network. Front Hum Neurosci 6:173. CrossRef Medline

Schubert T, Szameitat AJ (2003) Functional neuroanatomy of interference in overlapping dual tasks: an fMRI study. Cogn Brain Res 17:733-746. CrossRef

Shipstead Z, Redick TS, Engle RW (2012) Is working memory training effective? Psychol Bull 138: 628-654. CrossRef Medline

Stelzel C, Kraft A, Brandt SA, Schubert T (2008) Dissociable neural effects of task order control and task set maintenance during dual-task processing. J Cogn Neurosci 20:613-628. CrossRef Medline

Strobach T, Frensch PA, Soutschek A, Schubert T (2012) Investigation on the improvement and transfer of dual-task coordination skills. Psychol Res 76:794-811. CrossRef Medline

Szameitat AJ, Schubert T, Müller K, Von Cramon DY (2002) Localization of executive functions in dual-task performance with fMRI. J Cogn Neurosci 14:1184-1199. CrossRef Medline 
Szameitat AJ, Schubert T, Müller HJ (2011) How to test for dual-taskspecific effects in brain imaging studies-an evaluation of potential analysis methods. Neuroimage 54:1765-1773. CrossRef Medline

Takeuchi H, Sekiguchi A, Taki Y, Yokoyama S, Yomogida Y, Komuro N, Yamanouchi T, Suzuki S, Kawashima R (2010) Training of working memory impacts structural connectivity. J Neurosci 30:3297-3303. CrossRef Medline

Takeuchi H, Taki Y, Sassa Y, Hashizume H, Sekiguchi A, Fukushima A, Kawashima R (2011) Verbal working memory performance correlates with regional white matter structures in the frontoparietal regions. Neuropsychologia 49:3466-3473. CrossRef Medline

Thompson TW, Waskom ML, Garel KL, Cardenas-Iniguez C, Reynolds GO, Winter R, Chang P, Pollard K, Lala N, Alvarez GA, Gabrieli JD (2013)
Failure of working memory training to enhance cognition or intelligence. PLoS One 8:e63614. CrossRef Medline

van Turennout M, Ellmore T, Martin A (2000) Long-lasting cortical plasticity in the object naming system. Nat Neurosci 3:1329-1334. CrossRef Medline

von Bastian CC, Oberauer K (2014) Effects and mechanisms of working memory training: a review. Psychol Res 78:803-820. CrossRef Medline

Voytek B, Knight RT (2010) Prefrontal cortex and basal ganglia contributions to visual working memory. Proc Natl Acad Sci U S A 107:1816718172. CrossRef Medline

Ward BD (2000) Simultaneous inference for fMRI data. Available at: http:// afni.nimh.nih.gov/pub/dist/doc/manual/AlphaSim.pdf. 